Submitted то The Astrophysical Journal

Preprint typeset using $\mathrm{LAT}_{\mathrm{E}} \mathrm{X}$ style emulateapj v. 5/2/11

\title{
GALAXY CLUSTER BARYON FRACTIONS REVISITED
}

\author{
Anthony H. Gonzalez, ${ }^{1}$ Suresh Sivanandam, ${ }^{2,3}$ Ann I. Zabludoff, ${ }^{2}$ \& Dennis Zaritsky ${ }^{2}$ \\ Submitted to The Astrophysical Journal
}

\begin{abstract}
We measure the baryons contained in both the stellar and hot gas components for twelve galaxy clusters and groups at $z \sim 0.1$ with $M=1-5 \times 10^{14} \mathrm{M}_{\odot}$. This paper improves upon our previous work through the addition of XMM-Newton X-ray data, enabling measurements of the total mass and masses of each major baryonic component - intracluster medium, intracluster stars, and stars in galaxies — for each system. We recover a mean relation for the stellar mass versus halo mass, $M_{\star} \propto M_{500}^{-0.52 \pm 0.04}$, that is $1 \sigma$ shallower than our previous result. We confirm that the partitioning of baryons between the stellar and hot gas components is a strong function of $M_{500}$; the fractions of total mass in stars and Xray gas withing a sphere of radius $r_{500}$ scale as $f_{\star} \propto M_{500}^{-0.45 \pm 0.04}$ and $f_{\text {gas }} \propto M_{500}^{0.26 \pm 0.03}$, respectively. We also confirm that the combination of the brightest cluster galaxy and intracluster stars is an increasingly important contributor to the stellar baryon budget in lower halo masses. Studies that fail to fully account for intracluster stars typically underestimate the normalization of the stellar baryon fraction versus $M_{500}$ relation by $\sim 25 \%$. Our derived stellar baryon fractions are also higher, and the trend with halo mass weaker, than those derived from recent halo occupation distribution and abundance matching analyses. One difference from our previous work is the weak, but statistically significant, dependence here of the total baryon fraction upon halo mass: $f_{\text {bary }} \propto M_{500}^{0.16 \pm 0.04}$. For $M_{500} \gtrsim 2 \times 10^{14}$, the total baryon fractions within $r_{500}$ are on average $18 \%$ below the Universal value from the seven year WMAP analysis, or $7 \%$ below for the cosmological parameters from the Planck analysis. In the latter case the difference between the Universal value and cluster baryon fractions is less than the systematic uncertainties associated with the $M_{500}$ determinations. The total baryon fractions exhibit significant scatter, particularly at $M_{500}<2 \times 10^{14} \mathrm{M}_{\odot}$ where they range from $60-90 \%$, or $65-100 \%$, of the Universal value for WMAP7 and Planck, respectively. The ratio of the stellar-to-gas mass within $r_{500}\left(M_{\star} / M_{\text {gas }}\right)$, a measure of integrated star formation efficiency, strongly decreases with increasing $M_{500}$. This relation is tight, with an implied intrinsic scatter of $12 \%$. The fact that this relation remains tight at low mass implies that the larger scatter in the total baryon fractions at these masses arises from either true scatter in the total baryon content or observational scatter in $M_{500}$ rather than late-time physical processes such as redistribution of gas to beyond $r_{500}$. If the scatter in the baryon content at low mass is physical, then our results imply that in this mass range it is the integrated star formation efficiency and not the baryon fraction that is constant at fixed halo mass.

Subject headings: galaxies: clusters: general — galaxies:cD, formation, evolution, fundamental parameters - X-rays: galaxies: clusters
\end{abstract}

\section{INTRODUCTION}

Precision measurements of the total baryon content test the degree to which the expectation that clusters are nearly fair samples of the matter content of the Universe is valid (White \& Frenk 1991), and provide insight on the key physical processes that may act to drive observed total baryon fractions away from the Universal value (e.g., Nagai et al.|2007; McNamara \& Nulsen|2007, 2012; McCarthy et al. 2008, 2011; Simionescu et al. 2011, and references therein). How the baryons are distributed amongst the various components in a cluster also informs models for cluster assembly and cluster galaxy evolution.

\footnotetext{
${ }^{1}$ Department of Astronomy, University of Florida, Gainesville, FL 32611-2055

${ }^{2}$ Steward Observatory, University of Arizona, 933 North Cherry Avenue, Tucson, AZ 85721

${ }^{3}$ Dunlap Fellow, Dunlap Institute, University of Toronto, 50 St. George St, Toronto, ON, Canada

1 BASED ON OBSERVATIONS OBTAINED WITH XMMNEWTON, AN ESA SCIENCE MISSION WITH INSTRUMENTS AND CONTRIBUTIONS DIRECTLY FUNDED BY ESA MEMBER STATES AND NASA.
}

The partitioning of the baryons between the hot intracluster medium and stars constrains the net, integrated efficiency with which baryons are converted to stars in massive halos (hereafter star formation efficiency) and clarifies the importance of halo mass as a fundamental parameter tracing star formation efficiency Bryan 2000; Lin et al. 2003). This partitioning is also an important consideration for cosmological tests that aim to use the gas fraction to constrain $\Omega_{M}$ and $w$ (e.g., Allen et al. 2008), because variation will contribute to the observational scatter even for massive clusters. Finally, the partitioning of stellar baryons between satellite galaxies, the brightest cluster galaxy (BCG), and intracluster light provides a stringent test for models of cluster galaxy evolution and cluster luminosity function evolution, as the latter serves as a final reservoir for all stars liberated from other cluster galaxies (Conrov et al. 2007; Purcell et al. 2007; Behroozi et al. 2012; Watson et al. 2012).

In a pair of previous papers, we conducted an initial census of the stellar baryon content in a sample of 24 nearby systems with prominent BCGs (Gonzalez et al. 
2005, 2007, hereafter GZZ05 and GZZ07). In GZZ07 we used this sample in conjunction with published relations between $M_{500}$ and gas mass to quantify the total baryon fractions on group to cluster scales. Two central results were that the total baryon fraction within $r_{500}$ is roughly constant and $\sim 20 \%$ below the Universal value from WMAP (Komatsu et al. 2011), and that the gas and stellar baryon fractions have strong, opposite trends with system mass. The matching of the falling stellar and rising gas mass fractions with $M_{500}$ supports the suggestion made previously that the star formation efficiency decreases with increasing system mass (Bryan 2000; Lin et al. 2003). In GZZ07 we also emphasized the importance of including the intracluster light (ICL). Our observations demonstrated that the combination of the brightest cluster galaxy and intracluster light (hereafter BCG+ICL) dominate the stellar content within $r_{500}$ at group scales, but contain a decreasing percentage of the total stellar mass with increasing cluster mass. Omission of the ICL can thus bias both the slope and normalization of the derived relation between stellar and total mass.

Similar results of declining stellar mass fraction with halo mass have been obtained by a number of groups - albeit in most cases excluding the ICL. Power law slopes for $f_{*} \propto M^{\alpha}$ in the literature span the range $-0.65<\alpha<-0.25$ (e.g., Lin et al. 2003; Laganá et al. 2008; Giodini et al. 2009; Andreon 2010; Laganá et al. 2011; Zhang et al. 2011; Lin et al. 2012). While the case for a strong decrease of the stellar baryon fraction with increasing halo mass is compelling, there exists disagreement among these studies regarding the precise slopes of both the stellar and total baryon fractions as a function of cluster mass.

A significant contributing factor to the present unsettled state of the field is the quality of data available in past studies, including our own. To conduct a comprehensive study of the baryon fraction within a given radius (typically $r_{500}$ ), the data should include deep X-ray imaging for determination of the intracluster medium (ICM) gas mass, redshifts to determine cluster membership far below $L^{*}$, high-quality, multiband photometry suitable for both measurement of the ICL contribution and stellar masses of cluster members, and a reliable means of determining the cluster mass. The only existing study that satisfies all these criteria is Sanderson et al. (2013), which focuses upon a subset of the five lowest mass systems in the current sample and thus lacks the dynamic range in mass to robustly constrain the dependence of $f_{\star}$ upon $M_{500}$.

Our previous study (GZZ07) had the advantage of directly measuring the contribution of the ICL, a component that contains a significant percentage of the stellar baryons and becomes increasingly important with decreasing halo mass. However, a particularly critical limitation of this work was the lack of X-ray data. As a result, we constrained the total baryon fraction only by combining the derived stellar baryon fraction scaling relation with published X-ray data for a different cluster sample. This limitation also led us in GZZ07 to use cluster velocity dispersion $(\sigma)$ as a crude proxy for cluster mass. Specifically, we used data from Vikhlinin et al. (2006) to derive an $M_{X}-\sigma$ relation to convert from dispersion to $M_{500}$. The uncertainty associated with this relation is relevant because, as noted by Balogh et al.
(2008), if our mass determinations are systematically biased then the amplitude of the slope of the stellar baryon fraction scaling relation will also be biased. This limitation also precluded measuring scatter in the baryon fractions from cluster to cluster variation.

The central aim of this paper is to improve upon GZZ07, directly measuring the mass of each baryonic component plus the total cluster mass for a subset of twelve galaxy clusters from GZZ07. As part of this work, we also attempt to carefully assess the impact of any systematic biases that may impact any of the observed measurements. In section 2 we describe the cluster sample and data. Details of the total and gas mass measurements are provided in section [3, with a description of the stellar luminosity and stellar mass measurements in section 4. In section 5 we present our results for the partitioning of stars between the BCG+ICL and the galaxies, the partitioning of baryons between stars and gas, and the mass fractions for each component. We also consider the impact of potential systematics in this section and compare our results with previous studies. Section 6 presents the main conclusions from this work. Throughout the paper we adopt cosmological parameters consistent with the Komatsu et al. (2011) results from the seven-year Wilkinson Microwave Anisotropy Probe data $\left(H_{0}=70.2 \mathrm{~km} \mathrm{~s}^{-1}, \Omega_{M}=0.275, \Omega_{\Lambda}=0.725\right)$. Changes between the seven- and nine-year WMAP cosmological parameters (Bennett et al. 2012) have minimal effect on the results. We discuss the impact of the Planck cosmological parameters (Planck Collaboration et al. 2013) in section 5.3.4. We demonstrate that the main effect of the change in cosmology is to reduce the offset between the cluster baryon fractions and the Universal baryon mass fraction.

\section{CLUSTER SAMPLE}

The primary data set for this work consists of galaxy clusters drawn from the GZZ07 sample that have been observed by the $X M M-N$ ewton X-ray telescope (hereafter $X M M)$. A total of ten clusters initially satisfied this requirement. We exclude one of these clusters, Abell 3705, from our present analysis because the X-ray data indicate that it is unrelaxed with multiple X-ray peaks (Sivanandam et al. 2009). We also include three additional systems from GZZ07 that have recently been targeted with XMM by Sanderson et al. (2013) to explore the baryon fractions of low-mass systems, reanalyzing the data for consistency with our other systems.

In addition to the twelve clusters in our primary sample, we also analyze $X M M$ data for the most massive clusters from the Vikhlinin et al. (2006) sample to extend our mass baseline. While there are many massive clusters with published results in the literature, we opt here to only include ones from Vikhlinin et al. (2006) that we have reanalyzed to ensure that differences in analysis do not bias the observed trends. Specifically, we reprocess $X M M$ data for the three clusters from Vikhlinin et al. (2006) with $T>7.5 \mathrm{keV}$ (Abell 478, Abell 2029, and Abell 2390). While we lack the necessary data to constrain the stellar masses, we use these clusters to place a lower limit on the total baryon content at higher $M_{500}$ than are reached by our primary sample.

$X M M$ observations for clusters presented in this work were either taken through our guest observer (GO) pro- 
TABLE 1

X-ray Data Properties

\begin{tabular}{ccclllll}
\hline \hline Name & Obs ID $^{1}$ & \multicolumn{1}{c}{ Date } & Type $^{2}$ & Filter & $t_{\text {MOS }}(\mathrm{ks})$ & $\mathrm{t}_{\text {MOS,ff }}(\mathrm{ks})$ & Aperture $\left(^{\prime}\right)$ \\
\hline Abell 0122 & 0504160101 & 2007 Dec 03 & GO & Medium & $55.9 / 56.0$ & $48.5 / 49.4$ & $1.1-3.6$ \\
Abell 1651 & 0203020101 & 2004 Jul 01 & GO & Thin & $14.9 / 14.9$ & $8.2 / 8.3$ & $1.9-6.3$ \\
Abell 2401 & 0555220101 & 2010 Oct 29 & S12 & Thin & $56.8 / 56.9$ & $54.9 / 54.8$ & $1.6-5.3$ \\
Abell 2721 & 0201903801 & 2005 May 13 & XSA & Thin & $17.5 / 17.6$ & $9.5 / 9.9$ & $1.3-4.3$ \\
Abell 2811 & 0404520101 & 2006 Nov 28 & GO & Medium & $24.1 / 24.1$ & $21.7 / 22.0$ & $1.3-4.5$ \\
Abell 2955 & 0555220201 & 2008 Aug 02 & S12 & Thin & $81.4 / 81.5$ & $73.9 / 75.3$ & $1.0-3.3$ \\
Abell 2984 & 0201900601 & 2004 Dec 27 & XSA & Thin & $28.8 / 28.8$ & $27.9 / 27.5$ & $0.9-3.0$ \\
Abell 3112 & 0105660101 & 2000 Dec 24 & XSA & Medium & $23.1 / 23.1$ & $22.4 / 22.5$ & $1.7-5.8$ \\
Abell 3693 & 0404520201 & 2006 Oct 14 & GO & Medium & $34.2 / 34.3$ & $28.8 / 29.1$ & $0.9-3.2$ \\
Abell 4010 & 0404520501 & 2006 Nov 13 & GO & Thin & $19.4 / 19.4$ & $17.7 / 18.3$ & $1.3-4.3$ \\
Abell S0084 & 0201900401 & 2004 Dec 04 & XSA & Thin & $33.7 / 34.0$ & $18.3 / 18.1$ & $1.2-3.8$ \\
Abell S0296 & 0555220301 & 2008 Dec 26 & S12 & Thin & $68.8 / 68.9$ & $51.2 / 53.6$ & $1.3-4.5$ \\
\hline Abell 0478 & 0109880101 & 2002 Feb 15 & XSA & Thin & $69.7 / 125.0$ & $48.0 / 77.2$ & $1.9-6.4$ \\
Abell 2029 & 0551780201 & 2008 Jul 17 & XSA & Thin & $46.0 / 46.0$ & $32.7 / 33.4$ & $2.4-8.1$ \\
Abell 2390 & 0111270101 & 2001 Jun 19 & XSA & Thin & $22.2 / 22.2$ & $9.9 / 10.0$ & $1.1-3.5$ \\
\hline 1 XMM-Newton observation identification & & & & &
\end{tabular}

1 XMM-Newton observation identification.

${ }^{2}$ XSA denotes data from the XMM-Newton Science Archive; GO denotes data from our Guest Observer programs; S12 denotes systems from the Sanderson et al. (2012) program.

grams, drawn from Sanderson et al. (2013), or obtained from publicly available data in the XMM-Newton Science Archive (XSA). In Table 1, we present the details of the observations used in this work, including the observation IDs, time of observation, data source, filter choice, total integration time of the XMM EPIC-MOS camera observations both before and after flare-filtering, and the aperture used in the current analysis to derive cluster X-ray temperature. We present optical images of each cluster in our primary sample along with their associated Xray contours in Figure 1. The brightest cluster galaxy is nearly co-centric with the peak of the $\mathrm{X}$-ray emission, with offsets of less than $75 \mathrm{kpc}$ in all cases. A comparison of the outer $(3 \sigma)$ contours to $r_{500}$ (dashed circles) illustrate that the data typically reach beyond $0.7 r_{500}$ before the cluster emission drops below the background level.

\section{TOTAL AND GAS MASS FROM $X M M$}

We reduce the $X M M$ data using standard techniques for extended sources. Our cluster observations generally fill a large portion of the XMM field-of-view, requiring careful consideration of instrumental and cosmic backgrounds. For the processing we use $X M M$ Newton Science Analysis Software (SAS) version 12 with recent calibration data from September 2012. We use the XMM-ESAS software package to carry out flare filtering and determine the quiescent particle background (see Snowden et al. 2008, for full details on XMM backgrounds). We restrict our attention to the MOS1 and MOS2 cameras, as background estimation is more fully developed in XMM-ESAS for these than for the PN camera.

Our data analysis proceeds in two stages. The first stage consists of spectral fitting to determine $r_{500}$ and $M_{500}$. Given $r_{500}$, we then proceed to fit the surface brightness profiles for the $X M M$ data to determine the gas mass profile and total gas mass within $r_{500}$. We provide a brief description of our full procedure below.

\subsection{Basic Processing}

The data are flare-filtered for soft proton flares using the XMM-ESAS flare filtering technique. The final integration times after flare-filtering are listed in Table 1
In general, greater than $50 \%$ of the integration time of each cluster observation is used. However, this culling may not remove low-level soft proton flares, which need to be explicitly accounted for as part of the analysis (see 3.2). We next identify point sources, which must be masked prior to spectroscopic or spatial analysis of the extended cluster emission. For source detection we generate a combined $0.5-10 \mathrm{keV}$ joint MOS1 and MOS2 image and run the wavelet detection algorithm ewavelet with a $5 \sigma$ detection threshold on scales of $4-8$ pixels $\left(10^{\prime \prime}-20^{\prime \prime}\right)$. After using emldetect to determine the source extraction regions, we visually inspect the source extraction regions to ensure that there are no spurious source detections or sources associated with extended cluster emission. In the few cases where the detection algorithm flags cluster emission, we manually remove these detections from the point source catalog. This list is used to exclude regions in each observation when extracting spectra or generating a surface brightness map. Finally, we note that we exclude data from individual chips in instances where they exhibit anomalously elevated backgrounds in the $0.5-10 \mathrm{keV}$ image relative to the other chips. The excluded chips were typically CCD \#4 in MOS1 and CCD \#5 in MOS2.

\subsection{Spectral Fitting and $M_{500}$}

Because the sample is heterogenous in terms of depth, we choose a straight-forward and consistent method for determining $M_{500}$, which simply requires that the signalto-noise be sufficient to perform spectral fitting out to at least $0.5 r_{500}$. We use the Vikhlinin et al. (2009) calibration to convert from X-ray temperature measured within an aperture spanning 0.15 to $0.5 r_{500}$ to the total $M_{500}$ for each cluster. The relevant equations from Vikhlinin et al. (2009) are

$$
\begin{aligned}
T_{X} / T_{X, 2} & =0.9075+0.00625 T_{X, 2} \\
M_{500} & =M_{0}\left(T_{X} / 5 \mathrm{keV}\right)^{\alpha} E(z)^{-1}
\end{aligned}
$$

where $T_{X}$ and $T_{X, 2}$ are the X-ray temperatures measured at $r_{500}$ and $0.5 r_{500}$, respectively, $M_{0}=(3.02 \pm 0.11) \times$ $10^{14} h^{-1} M_{\odot}, \alpha=1.53 \pm 0.08$, and $E(z)$, which describes the evolution of the Hubble parameter, is defined as in (Peebles 1993, pp. 310-321). 

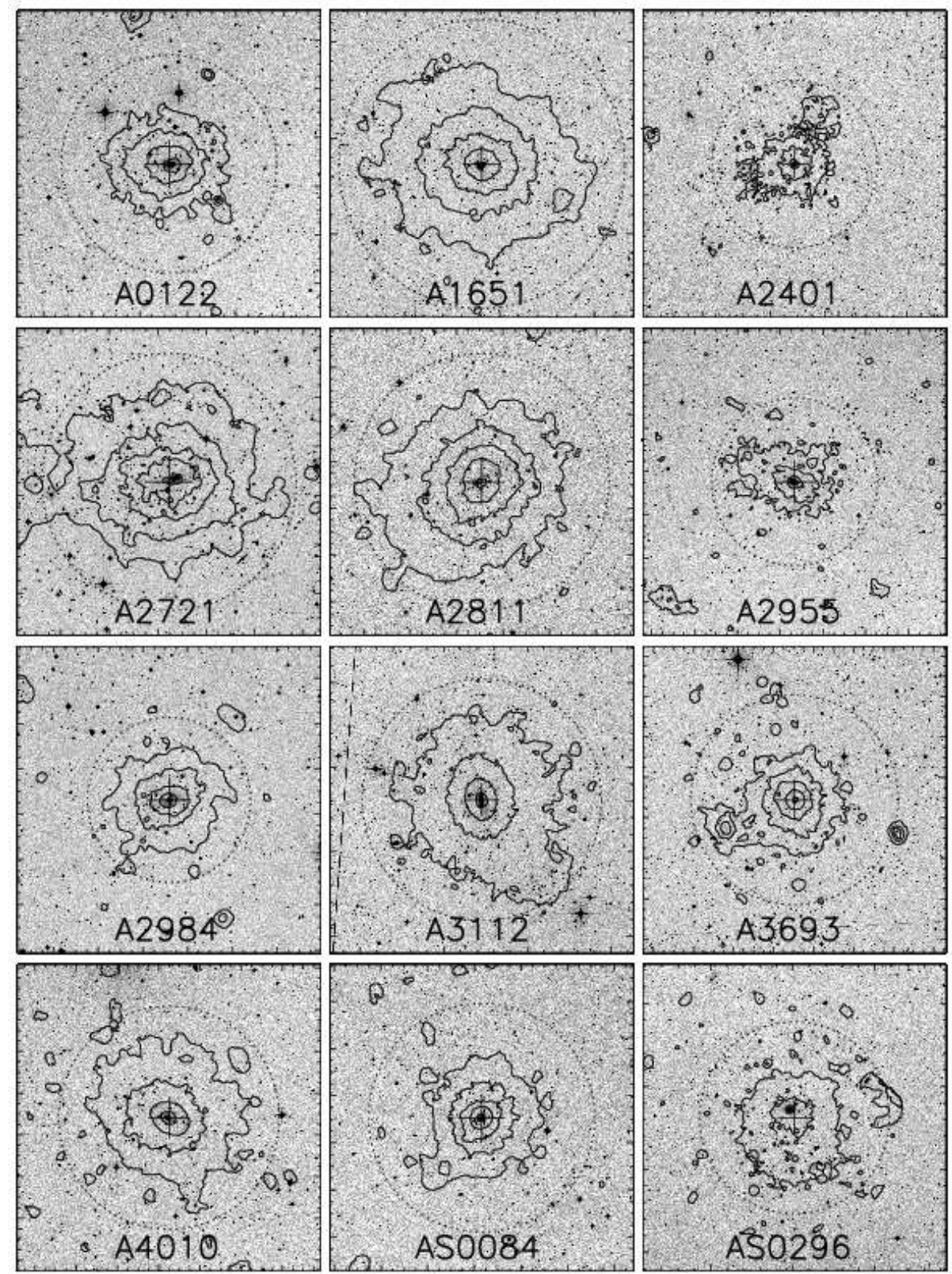

FIG. 1. - $2.5 \times 2.5 \mathrm{Mpc}$ thumbnails from the Digitized Sky Survey (DSS) for the primary sample of twelve clusters, with their X-ray surface brightness contours overlaid. The X-ray contours are generated from an adaptively smoothed surface brightness map. There are five contour levels which are logarithmically spaced at equal intervals. The lowest one corresponds to a value $3 \sigma$ above the brightness level measured at the outskirts of the XMM EPIC-MOS field-of-view, and the highest value corresponds to 0.1 dex below the brightest value. The crosshair indicates the fitted centre for the X-ray emission. The dotted circle is the measured $r_{500}$ value for each cluster.

Measuring $T_{X}$ is an iterative process where we first estimate $r_{500}$ to compute the appropriate spectral extraction aperture, and iterate until the computed value of $r_{500}$ is stable. The spectra are extracted using the $X M M$ $E S A S$ software and fit using XSPEC 12. XMM-ESAS generates the appropriate quiescent particle background spectrum, which is then subtracted from the spectra before fitting. The fit model and method used for the spectral fitting is outlined in Snowden et al. (2008) and is identical to the one described in our previous work (Sivanandam et al. 2009). The fit function properly accounts for instrument background such as residual soft proton flux and fluorescent lines, the cosmic background, and the thermal emission from the cluster ICM. Only the spectrum within the extracted aperture is fit along with
ROSAT All-Sky Survey X-ray background data to constrain the cosmic background. The initial guess of $r_{500}$ is computed using the temperature determined from an aperture with inner and outer radii of $0^{\prime}$ and $6^{\prime}$, respectively. The spectral extraction and fits are repeated until the inner and outer extraction radii do not change by more than $10^{\prime \prime}$. The extraction aperture for each cluster are given in Table 1 and the final derived $T_{X, 2}$ are listed in Table 3 . The $1 \sigma$ uncertainties are determined by carrying out 10,000 Monte Carlo simulations, which take into account the uncertainties in $T_{X, 2}$ and the Vikhlinin et al. (2009) fit functions. The derived $M_{500}$ values are also given in Table 3 .

\subsection{Surface Brightness Profile Fitting and Gas Mass}




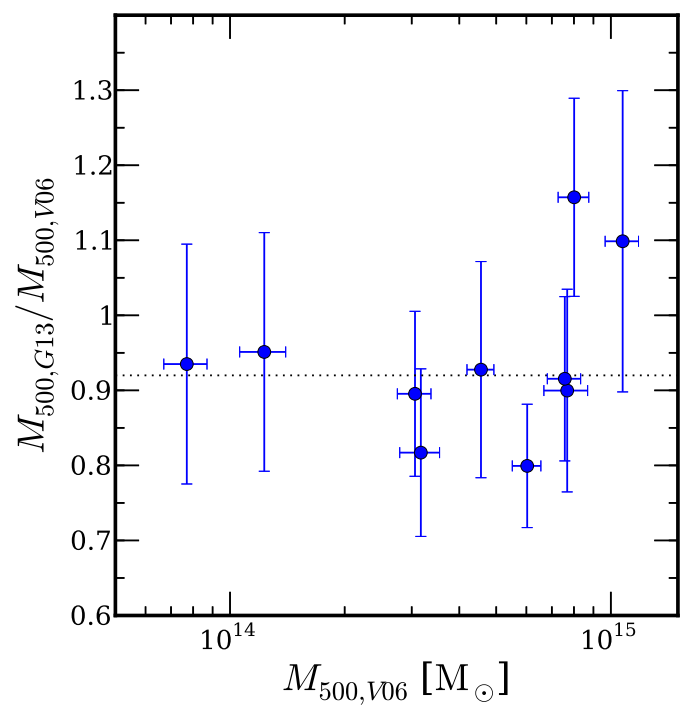

FIG. 2.- Comparison of $M_{500}$ measurements for a common set of clusters as measured by Vikhlinin et al. (2006, V06) using Chandra data with our $X M M$-derived values (G13). Our $M_{500}$ values are on average $8 \%$ below those of Vikhlinin et al. (2006, dotted line), with no observable dependence upon cluster mass.

We compute the gas mass within $r_{500}$ using the gas density profile determined from the imaging and spectral information. We use the same methods described in Sivanandam et al. (2009) with a few minor refinements. The technique involves fitting a radially symmetric projected $\beta$ profile (Cavaliere \& Fusco-Femiano 1978) to an $\mathrm{X}$-ray image of the cluster to determine the structural parameters of the gas density profile, i.e., $r_{0}$ and $\beta$. The central gas density is determined using the cluster model's normalization (norm) parameter obtained from the XSPEC spectral fits within a given radial bin combined with the structural parameters. One change from our previous analysis is that we now use the $0.4-1.25$ $\mathrm{keV}$ X-ray images for the surface brightness fits rather than a simultaneous fit to the $0.4-1.25 \mathrm{keV}$ and $2-7.2$ $\mathrm{keV}$ images. As some of the clusters have cool-cores, this approach minimizes the temperature dependence of the structural parameters. We also model the instrumental background component slightly differently. We include a flat instrumental background, which is set to be the average quiescent particle background as determined by the $X M M-E S A S$ software. The instrument background is held fixed during the initial fit, which determines the projected $\beta$ model parameters and the cosmic background contribution. After the initial fit converges, a new fit is carried out where the value of the instrument background is allowed to vary. This freedom is necessary to account for residual soft proton flux not removed during flare filtering. With these final fit parameters, the $\beta$ model is integrated out to $r_{500}$ to obtain $M_{g a s, 500}$. Fit parameters and calculated properties are listed in Table 3

\subsection{Comparisons with other measurements}

We next check the consistency of our $M_{500}$ and gas fraction $\left(f_{\text {gas }}\right)$ measurements with other studies. Because the $M_{500}-T_{X}$ relation from Vikhlinin et al. (2009) was calibrated using Chandra data, and there may exist slight offsets between $X M M$ and Chandra temperatures (Nevalainen et al. 2010), we compare our $M_{500}$

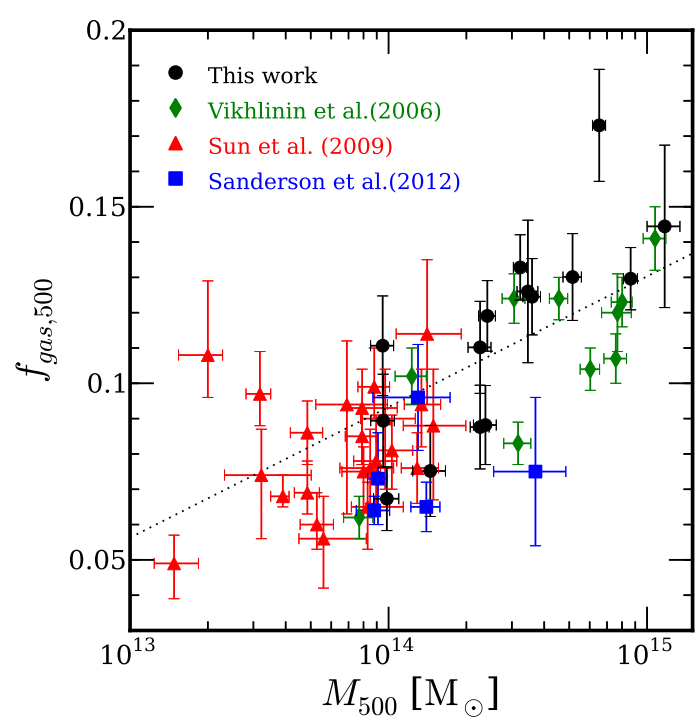

FIG. 3. - X-ray gas fraction within $r_{500}$ as a function of $M_{500}$. Different data sets are shown as a comparison with our work (black circles). The red triangles are from a Chandra study of groups (Sun et al. 2009), while the green diamonds are Chandra measurements of clusters by Vikhlinin et al. (2006). We also include as boxes the measurements from Sanderson et al. (2013) for five systems in our sample. The dotted line is the fit to the gas fraction as determined by Vikhlinin et al. (2009). The consistency of X-ray and optical centers, and regularity of the X-ray countours, argue that these systems are roughly relaxed, although small X-ray substructures are present in several. At low mass our gas fractions span a similar range to those from Sun et al. (2009). At higher mass our measurements are consistent with the mean trend, but are $\sim 10 \%$ higher than the Vikhlinin et al. (2006) data. This offset is consistent with arising simply from the normalization difference for $M_{500}$ shown in Figure 2

with $M_{500}$ measured using Chandra data(Vikhlinin et al. 2006) for a common set of clusters. The result of our comparison is shown in Figure 2. While the majority of clusters are consistent to within the statistical errors, the $X M M$ masses are on average $8 \%$ lower. This offset, which is likely the result of differences in the cross-calibration of Chandra and XMM (Nevalainen et al. 2010), provides an estimate of the systematic uncertainty in $M_{500}$ from instrumental effects. In section 5.4.2 we also consider the potential systematic uncertainty associated with the use of hydrostatic gas masses.

We also compare our $f_{\text {gas }}$ with other group and cluster samples (Figure 31). We compile these measurements from three different samples, Vikhlinin et al. (2006), Sun et al. (2009), and Sanderson et al. (2013). The two Chandra studies, (Sun et al. 2009) and Vikhlinin et al. (2006), together cover the full mass range from groups to clusters. We find that our measurements are consistent with the mean trend, but systematically offset to higher gas fractions by $\sim 10 \%$ relative to the Vikhlinin et al. (2006) data (see 5.3.1). The observed offset is consistent with arising simply from the normalization difference for $M_{500}$ shown in Figure 2, At low mass, our gas fractions span a similar range to those from Sun et al. (2009).

\section{LUMINOSITY AND STELLAR MASS DETERMINATIONS}

4 We note that while our $M_{500}$ values are derived using an integrated temperature measurement, Vikhlinin et al. (2006) use gas density and temperature profiles that extend out to $r_{500}$ to directly estimate hydrostatic masses for these systems within that radius. 


\subsection{Luminosities}

For each component, we compute the total luminosity within $r_{500}$ using the radius derived from the $X M M$ data. To compute the luminosities of the BCG+ICL and cluster galaxy population, we use the same data and techniques as in GZZ05 and GZZ07. As described in GZZ05, all imaging was obtained in Gunn $i$, with photometry calibrated to Cousins I using Landolt standards. In converting from magnitudes to luminosities, we take the absolute magnitude of the Sun to be $M_{I}=4.09$ (Vega; Mancone \& Gonzalez 2012) 5 which is fainter than the normalization, $M_{I}=3.94$, used in GZZ07. This change in normalization results in all luminosities - and hence stellar masses - increasing by $15 \%$ relative to GZZ07, and corrects an inconsistency with Cappellari et al. (2006) in our previous paper. The luminosities for the BCG+ICL component are computed using the same models, and the resulting values are typically identical to those quoted in Table 1 of that paper, modulo the change in $M_{I}$, because the physical extent of this component is generally much smaller than $r_{500}$. Error bars are computed by propagating the uncertainties in the model fit to the BCG+ICL component.

We compute the total luminosity of the galaxy population, excluding the BCG+ICL, using statistical background subtraction. Specifically, we compute the total flux from galaxies with $m_{B C G}<m_{I}<18$ within $r_{500}$, and use a $30^{\prime}-60^{\prime}$ annular aperture encircling the cluster to compute a statistical background correction for subtraction. In GZZ07 we assessed the potential impact of large-scale structure on the derived background corrections via comparisons with the SDSS luminosity function from Blanton et al. (2003), finding it to be minimal. We next convert from flux to absolute magnitude using the cluster redshift, and apply a completeness correction to account for galaxies with $m_{I}>18$. For this correction we adopt the cluster luminosity function of Christlein \& Zabludoff (2003), using $R-I=0.82$ to convert between filters. Additional details of this procedure are discussed in Gonzalez et al. (2007). The two dominant uncertainties in the total galaxy luminosity are uncertainty associated with the statistical background subtraction and systematic uncertainty assocated with the completeness correction for the faint end of the luminosity function. As noted in GZZ07, the derived luminosity is $12 \%$ lower if one assumes $\alpha=-1$ instead of $\alpha=-1.21$. No strong dependence of the faint end slope upon mass is expected even at group scales (e.g. Zandivarez \& Martínez 2011), and so this assumption should yield no scale-dependent bias. We therefore estimate a total uncertainty of $0.15 \mathrm{mag}$ for the integrated magnitude of the cluster galaxy population. The derived luminosities and associated uncertainties for the BCG+ICL and the total luminosity of the system are included in Table 3 .

\subsection{Stellar Mass}

Conversion of observed luminosity to stellar mass requires choosing an appropriate stellar mass-to-light ratio $\left(\Upsilon_{\star}\right)$ for the cluster galaxy population. While in principle straightforward, in practice this is the least well

5 Based upon the solar spectrum

TABLE 2

Derived Mass Fractions $\left(r<r_{500}\right)$

\begin{tabular}{cccc}
\hline \hline Cluster & $f_{\text {gas }}$ & $f_{\text {stellar }}$ & $f_{\text {baryons }}$ \\
& & & \\
\hline Abell 0122 & $0.088 \pm .012$ & $0.024 \pm .002$ & $0.112 \pm .012$ \\
Abell 1651 & $0.130 \pm .012$ & $0.013 \pm .001$ & $0.143 \pm .012$ \\
Abell 2401 & $0.089 \pm .013$ & $0.028 \pm .003$ & $0.118 \pm .014$ \\
Abell 2721 & $0.126 \pm .020$ & $0.017 \pm .002$ & $0.143 \pm .020$ \\
Abell 2811 & $0.125 \pm .011$ & $0.013 \pm .002$ & $0.138 \pm .011$ \\
Abell 2955 & $0.067 \pm .010$ & $0.030 \pm .004$ & $0.097 \pm .011$ \\
Abell 2984 & $0.111 \pm .014$ & $0.041 \pm .005$ & $0.152 \pm .015$ \\
Abell 3112 & $0.133 \pm .009$ & $0.022 \pm .002$ & $0.155 \pm .009$ \\
Abell 3693 & $0.110 \pm .013$ & $0.023 \pm .003$ & $0.133 \pm .013$ \\
Abell 4010 & $0.119 \pm .010$ & $0.023 \pm .003$ & $0.143 \pm .010$ \\
Abell S0084 & $0.088 \pm .011$ & $0.022 \pm .003$ & $0.110 \pm .011$ \\
Abell S0296 & $0.075 \pm .013$ & $0.020 \pm .003$ & $0.095 \pm .013$ \\
\hline Abell 0478 & $0.173 \pm .016$ & - & - \\
Abell 2029 & $0.130 \pm .009$ & - & - \\
Abell 2390 & $0.144 \pm .023$ & - & -
\end{tabular}

Note. - The quoted stellar baryon fractions include a deprojection correction, as discussed in the text.

constrained input when computing stellar baryon fractions. There are several approaches that one can take for this conversion, as we discuss in $\$ 5.4 .1$.

We choose to base our estimate for $\Upsilon_{\star}$ on the dynamical results from Cappellari et al. (2006), as in GZZ07. The strength of this approach is that it avoids the use of stellar population models and associated systematic uncertainties. There are however several limitations. First, the population of galaxies with robust dynamical measurements of central mass-to-light ratios is predominantly comprised of quiescent galaxies, which have systematically higher $\Upsilon_{\star}$ values than the star-forming population. In GZZ07 we ignored the impact of the star-forming population in altering the global mass-tolight ratio. We retain in this paper the assumption of a purely passive population, as in GZZ07, but assess the impact of this assumption in \$5.4.1. Second, in the Cappellari et al. (2006) study the derived dynamical masses within the effective radius were noted to include $0-30 \%$ dark matter contributions, indicating that the stellar $\Upsilon_{\star}$ values are smaller by a corresponding amount. While the contribution of dark matter in Cappellari et al. (2006) can be up to $30 \%$ for individual systems, it is generally less for the slow rotators that best represent the most massive galaxies, and contribute substantially to the total baryon fraction. In this paper we assume an average contribution of $15 \%$ dark matter, a change from GZZ07 in which we assumed a 0\% contribution. The total impact of these limitations is expected to be minor, as we discuss further in $\$ 5.4 .1$.

Cappellari et al. (2006) provide an empirical determination of $\Upsilon_{\star}$ using Schwarzschild dynamical modelling of two-dimensional kinematic data from SAURON. Equation (9) in Cappellari et al. (2006) quantifies the luminosity dependence of $\Upsilon_{\star}$ in the $I$-band, which is consistent with more recent observations from ATLAS3D (Cappellari, priv. comm). We use a similar method as in GZZ07, computing a luminosity-weighted $\Upsilon_{\star, I}$ for $L>0.25 L_{*}$ (the range over which the SAURON relation is established) and using the same Christlein \& Zabludoff (2003) luminosity function. We then include a correction for the estimated $15 \%$ dark matter contribution, deriv- 
TABLE 3

Observed Cluster Properties

\begin{tabular}{|c|c|c|c|c|c|c|c|c|c|}
\hline Cluster & $z$ & $\begin{array}{l}T_{X, 2} \\
(\mathrm{keV})\end{array}$ & $\begin{array}{c}L_{B C G+I C L} \\
\left(10^{12} \mathrm{~L} \odot\right)\end{array}$ & $\begin{array}{c}L_{\text {Total }} \\
\left(10^{12} \mathrm{~L}_{\odot}\right)\end{array}$ & $\begin{array}{c}r_{500} \\
(\mathrm{Mpc})\end{array}$ & $\begin{array}{c}M_{500} \\
\left(10^{14} \mathrm{M}_{\odot}\right)\end{array}$ & $\begin{array}{c}M_{g a s, 500} \\
\left(10^{13} \mathrm{M}_{\odot}\right)\end{array}$ & $\begin{array}{l}M_{\star, 2 D, 500} \\
\left(10^{13} \mathrm{M}_{\odot}\right)\end{array}$ & $\begin{array}{l}M_{\star, 3 D, 500} \\
\left(10^{13} \mathrm{M}_{\odot}\right)\end{array}$ \\
\hline 0122 & 134 & $365+0$ & $84+1>0$ & $2.57 \pm 0$ & $9 \pm .03$ & \pm .19 & $198+21$ & $0.68 \pm .04$ & $0.55 \pm .03$ \\
\hline & 845 & .25 & 009 & & $8 \pm .03$ & \pm .42 & $670+32$ & $0.82 \pm .06$ & \pm .05 \\
\hline Abell 2401 & 0.0571 & $2.06 \pm 0.07$ & $.33 \pm 0.01$ & $1.30 \pm 0.09$ & $0.68 \pm .02$ & $0.95 \pm .10$ & $0.85 \pm .09$ & $0.35 \pm .03$ & $0.27 \pm .02$ \\
\hline Abell 2721 & 0.1144 & $4.78 \pm 0.23$ & $57+001$ & $2.81 \pm 0.21$ & $1.03 \pm .03$ & $3.46 \pm .32$ & & $0.74 \pm .06$ & $0.57 \pm .04$ \\
\hline Abell 2811 & 1079 & $4.89 \pm$ & 4 & 2.13 & $1.04 \pm .03$ & .28 & .17 & $0.56 \pm .05$ & .04 \\
\hline Abell 2955 & 0.0943 & $2.13 \pm 0.10$ & $60 \pm 0.03$ & $1.33 \pm 0.08$ & $0.68 \pm .04$ & $0.99 \pm .11$ & $0.66 \pm .05$ & $0.35 \pm .02$ & $0.30 \pm .02$ \\
\hline Abell 2984 & 1042 & $2.08 \pm 0.07$ & $86 \pm 0.03$ & $1.72 \pm 0.08$ & $0.67 \pm .01$ & $0.95 \pm .10$ & & $0.46 \pm .02$ & $0.39 \pm .02$ \\
\hline Abell 3112 & 0.0750 & $4.54 \pm 0.11$ & $0.93 \pm 0.05$ & $3.33 \pm 0.23$ & $1.02 \pm .02$ & $3.23 \pm .19$ & $4.29 \pm .16$ & $0.88 \pm .06$ & $0.70 \pm .04$ \\
\hline Abell 3693 & 1237 & $3.63 \pm 0.20$ & $71+0 \Omega$ & $2.42 \pm 0.18$ & $=.03$ & $2.26 \pm .23$ & & $0.64 \pm .05$ & $0.51 \pm .04$ \\
\hline Abell 4010 & 0.0963 & $3.78 \pm 0.13$ & $.81 \pm 0.12$ & $2.65 \pm 0.21$ & $0.92 \pm .02$ & \pm .18 & $2.87 \pm .11$ & $0.70 \pm .06$ & $0.56 \pm .05$ \\
\hline Abell S0084 & 0.1100 & $3.75 \pm 0.20$ & $0.70 \pm 0.02$ & $2.46 \pm 0.16$ & $0.91 \pm .03$ & $2.37 \pm .24$ & $2.09 \pm .16$ & $0.65 \pm .04$ & $0.52 \pm .03$ \\
\hline Abell S0296 & 0.0696 & $2.70 \pm 0.21$ & $0.57 \pm 0.01$ & $1.30 \pm 0.07$ & $0.78 \pm .04$ & $1.45 \pm .21$ & $1.09 \pm .10$ & $0.35 \pm .02$ & $0.29 \pm .01$ \\
\hline Abell 0478 & 0.0881 & $7.09 \pm 0.12$ & $=$ & $=$ & $1.28 \pm 0.03$ & $6.58 \pm 0.38$ & $11.5 \pm 0.8$ & & \\
\hline Abell 2029 & 0.0773 & $8.41 \pm 0.12$ & - & - & $1.42 \pm 0.03$ & $8.71 \pm 0.55$ & $12.0 \pm 0.4$ & 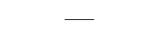 & - \\
\hline Abell 2390 & 0.2329 & $10.6 \pm 0.8$ & - & - & $1.50 \pm 0.07$ & $11.8 \pm 1.8$ & $17.2 \pm 1.0$ & - & - \\
\hline
\end{tabular}

Note. - Abell 0478, Abell 2029, and Abell 2390 are not part of the main sample. These clusters were included only in the X-ray analysis to extend the baseline to higher mass, but have no photometry equivalent to the other systems with which to measure the stellar mass. At the high mass end; however, the stellar component contributes a relatively small fraction of the total baryons. The luminosities include appropriate e+k corrections for each galaxy from GZZ07. The stellar masses are quoted as observed, with no deprojection correction applied.

ing $\Upsilon_{\star, I}=2.65$. This value lies between the median $\Upsilon_{\star}$ derived for Chabrier and Salpeter IMFs via stellar population modelling in Leauthaud et al. (2012), and is $26 \%$ lower than the value used in GZZ07 due to the combination of the dark matter correction and a correction to our previous $\Upsilon_{\star}$ calculation. We note that the resultant stellar masses are however only $\sim 13 \%$ lower than those used in our previous work because the dark matter correction is offset by the change in the absolute magnitude of the Sun between the two papers. We use same the $e+k$ corrections as in GZZ07 to account for passive evolution between the redshifts of our clusters and that of the Cappellari et al. (2006) sample, which lie at $z<0.01$.

One final consideration in deriving stellar mass is line of sight projection. While the derived gas and total masses are three-dimensional quantities, we measure the projected stellar mass within $r_{500}$. In most previous studies, including GZZ07, no attempt was made to deproject the stellar mass and derive an estimate of the total stellar mass enclosed within a three-dimensional sphere of radius $r_{500}$. 6 For the current paper we compute both the observed and deprojected stellar masses, hereafter $M_{\star, 2 D}$ and $M_{\star, 3 D}$, respectively. We calculate $M_{\star, 3 D}$ using a similar approach to Sanderson et al. (2013). Specifically, we model the galaxy distribution using an NFW profile with $c=2.9$ (Lin et al. 2004). For this concentration, $71 \%$ of the galaxy stellar mass within a projected $r_{500}$ lies within a sphere or radius $r_{500}$. We apply no deprojection correction to the $\mathrm{BCG}+\mathrm{ICL}$, as the observed physical extent of the ICL is significantly less than $r_{500}$ in all cases. This deprojection correction decreases the normalization of the stellar baryon fraction relation by $\sim 20 \%$. In addition, because the importance of the galaxy population relative to the BCG+ICL increases with $M_{500}$, this correction has the greatest impact at high mass and also acts to steepen the relation. We list both the projected and deprojected stellar masses in Table 3.

${ }^{6}$ An exception is Giodini et al. (2009), in which the authors do apply a deprojection correction.

\section{RESULTS AND DISCUSSION}

\subsection{Stripping Efficiency: Partitioning of Stars between the $B C G+I C L$ and Galaxies}

Given the updated $M_{500}$ and $r_{500}$ determinations, we first investigate what percentage of the cluster luminosity is contained within the central BCG and ICL as a function of $M_{500}$. In GZZ07 we found a strong trend of the BCG+ICL contributing a decreasing percentage of the total cluster luminosity with increasing cluster velocity dispersion. In Figure 4 we show an updated version of this relation, comparing the original results with luminosity fractions calculated within the $r_{500}$ radii derived from the X-ray data. The main change is a modest flattening of the trend, as the percentages have decreased for the lower dispersion systems and increased for the highest mass systems. A fit to the data still however yields $L_{B C G+I C L} / L_{\text {Total }} \propto \sigma^{-0.78 \pm 0.12}$ for $r<r_{500}$ within the range of dispersions covered by this sample.

With the inclusion of the X-ray data, we can now also move beyond velocity dispersion, which is a high-scatter proxy for cluster mass, and directly investigate the massdependence of the fractional BCG+ICL contribution. In Figure 5 we plot the proportion of the total luminosity within $r_{500}$ that is contained in the BCG+ICL as a function of $M_{500}$. The trend in fractional BCG+ICL contribution originally observed with velocity dispersion in GZZ07 is seen with $M_{500}$ at comparable statistical significance, with $L_{B C G+I C L} / L_{\text {Total }} \propto M_{500}^{-0.37 \pm 0.06}$. This trend has also been confirmed independently by searches for intracluster suernovae (McGee \& Balogh 2010; Sand et al. 2011). Low mass systems in our sample exhibit a substantially higher percentage of stars in the BCG+ICL than the most massive systems, with $40-50 \%$ of the stellar luminosity contained in this component for $M_{500} \approx 1 \times 10^{14} \mathrm{M}_{\odot}$. There is, however, one outlier (A2955) at low mass and low BCG+ICL content from the Sanderson et al. (2013) sample. While it is difficult to draw strong conclusions based upon a single data point, this group may be indicative of significant scatter in the partitioning of stars between $\mathrm{BCG}+\mathrm{ICL}$ and galaxies at 


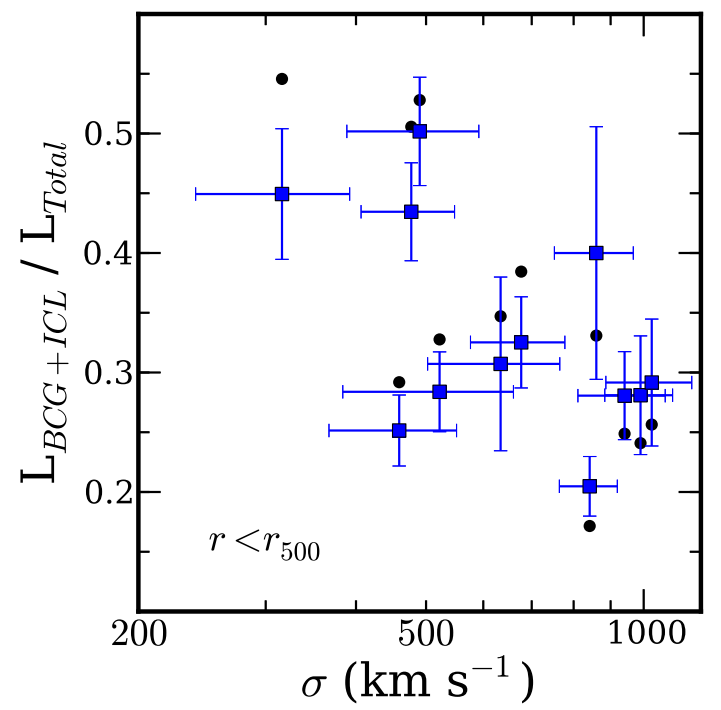

FIG. 4.- Contribution of light within $r_{500}$ contained by the BCG+ICL as function of cluster velocity dispersion. The black circles denote the values from GZZ07; the blue squares correspond to the revised contribution based upon the new $r_{500}$ values from our current work, including the groups from Sanderson et al. (2012).

low mass.

\subsection{Star Formation Efficiency: Partitioning of the Stars and Gas}

An important strength of the current data set is that it enables us to compare the stellar and gaseous masses for individual systems, thereby directly assessing how the efficiency with which baryons are converted to stars depends upon cluster mass, as well as cluster-to-cluster scatter about the mean trend. Zhang et al. (2011) explore this question, albeit without inclusion of the ICL, using XMM and ROSAT data in combination with SDSS photometry. The authors find a relatively strong dependence, $M_{\star, 2 D} / M_{\text {gas }} \propto M_{500}^{-0.537 \pm 0.101}$, within $r_{500}$ with an intrinsic (physical) scatter of $29 \pm 5 \%$. In Figure 6 we present our measurement of this ratio, along with a comparison to the best-fit relation from Zhang et al. (2011), which assumed a Salpeter IMF and used separate $\Upsilon_{\star}$ for the star-forming and quiescent populations. For a program such as Zhang et al. (2011), which uses SDSS photometry for the BCG, we expect that $\sim 60 \%$ of the total light in the BCG+ICL will be missed 7 We therefore also include points for which we have removed $60 \%$ of the $\mathrm{BCG}+\mathrm{ICL}$ stellar mass in our systems to enable a more direct comparison of slopes between the two data sets. We define this as the "50 kpc" case below. Residual differences will be present in the normalizations due to the different treatments of the IMF and star-forming versus quiescent populations, as well as to known biases in SDSS photometry for brightest cluster galaxies (Lauer et al. 2007; Bernardi et al. 2007; Hyde \& Bernardi 2009).

7 Gonzalez et al. (2005) find that a $50 \mathrm{kpc}$ aperture contains $\sim 40 \%$ of the total light in the BCG+ICL. Magnitudes measured within this aperture are close to the Kron total magnitudes derived for BCGs (e.g., Stott et al. 2010, found consistency to within 0.05 $\mathrm{mag}$ ), so a $60 \%$ fractional reduction in the total luminosity should be a reasonable estimate.

8 It has been pointed out by multiple authors that the SDSS magnitudes, such as those used by Zhang et al. (2011), are un-

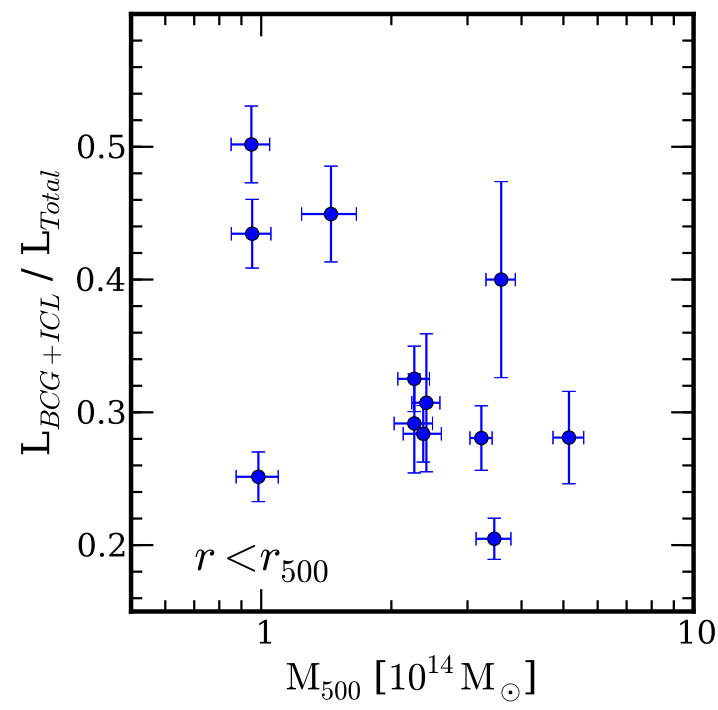

FIG. 5.- Fraction of light within $r_{500}$ contained by the BCG+ICL as function of cluster mass. For the majority of low mass systems the BCG+ICL contribution to the total luminosity is nearly comparable to that of the galaxy population.

If we only include the stellar baryons in the cluster galaxy population and the BCG+ICL contribution within the inner $50 \mathrm{kpc}$ (red, square data points), then we recover a slope, $-0.79 \pm 0.05$, which is approximately $2.5 \sigma$ steeper than Zhang et al. (2011). Inclusion of the ICL beyond $50 \mathrm{kpc}$ slightly steepens this slope to $-0.78 \pm 0.04$ due to the larger fractional contribution of the ICL at lower mass. The best fit relation is presented in Table 4 along with the best fit for $M_{\star, 3 D} / M_{\text {gas }}$, which has a comparable slope.

The normalization of the relation confirms the claim in GZZ07 that the stellar component contributes nearly half of the total baryonic mass by group scales. The $17 \%$ scatter about the best fit relation can be explained by a combination of the known observational uncertainties and $12 \%$ intrinsic scatter. The physical implication of this low intrinsic scatter is that star formation efficiency at a fixed cluster mass is quite uniform.

\subsection{Stellar, Hot Gas, and Total Baryon Mass Fractions}

The combination of X-ray and optical data described above enable us to conduct the first analysis of the baryon mass fractions within $r_{500}$ for a statistical sample of clusters in which each system has direct determinations of the stellar, intracluster, and dark matter contributions within comparable radii.

\subsubsection{Gas Mass Fraction}

We fit the relation $M_{\text {gas }}=a M_{500}^{b}$, where $M_{\text {gas }}$ is the gas mass within $r_{500}$ using orthogonal distance regression. We list the best fit parameters in Table 4. The gas fractions derived in this paper yield a mass dependence slightly steeper than the fiducial relation derived from Vikhlinin et al. (2006) that was used in GZZ07.

derestimates due to oversubtraction of the sky in crowded regions and for extended sources (Lauer et al. 2007; Bernardi et al. 2007; [Hyde \& Bernardi 2009), with Hyde \& Bernardi (2009) finding magnitude corrections of up to $30 \%$. We make no attempt here to exclude additional luminosity in our systems to match this effect in the SDSS photometry. 

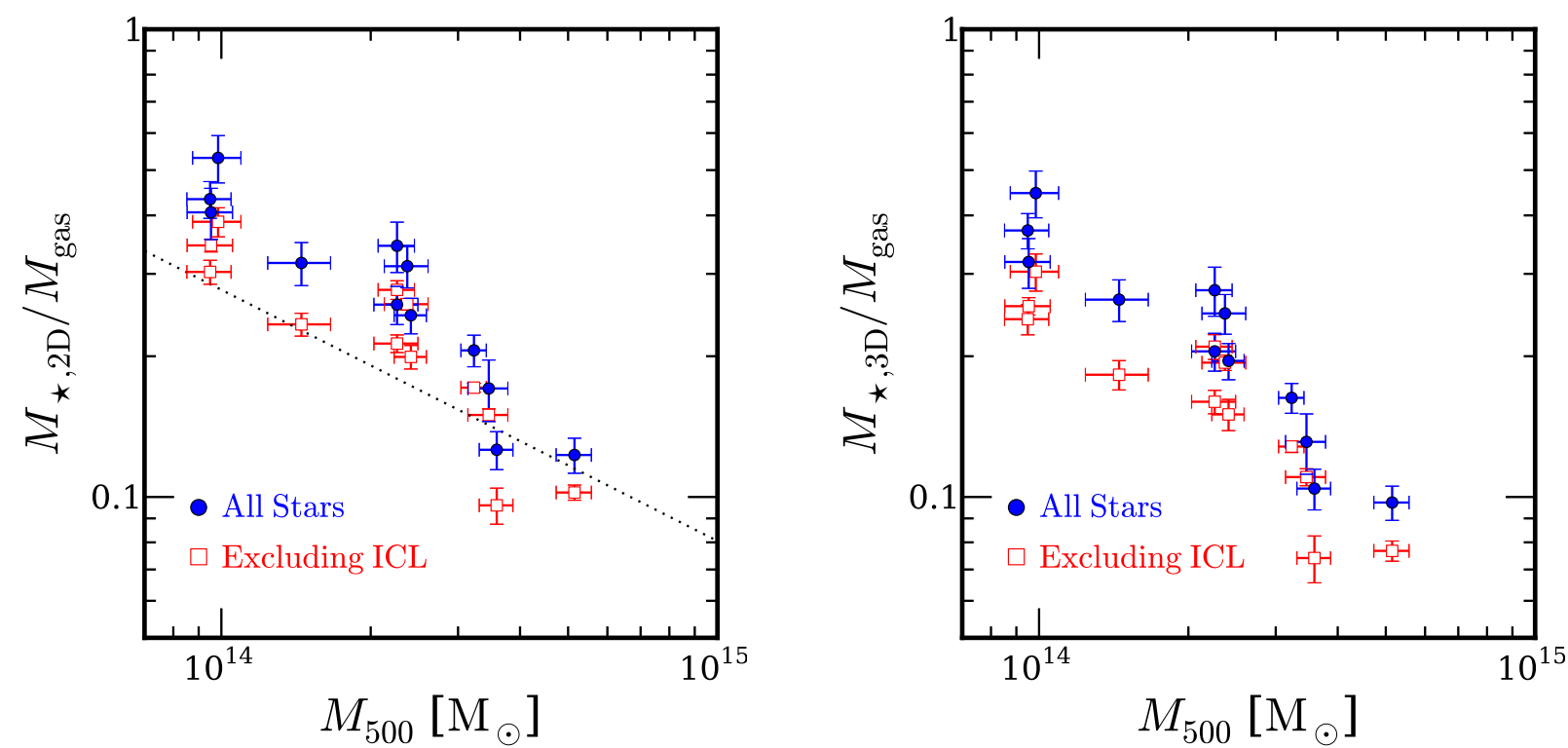

FIG. 6.- Ratio of stellar to gas mass as a function of $M_{500}$. The left panel shows the total stellar mass projected within $r_{500}$, while the right panel includes a deprojection correction (see $\$ 5.3 .2)$. We plot this ratio for both the total stellar mass (BCG+ICL and cluster galaxies, blue filled circles) and the stellar mass contained only in the cluster galaxies and the BCG, excluding the ICL (red open squares). For comparison, we overplot as a dotted line the relation (with no deprojection correction) derived by Zhang et al. (2011), which did not include the ICL and assumed a Salpeter IMF in deriving stellar masses. Zhang et al. (2011) also did not apply a deprojection correction, so the lefthand panel is most directly comparable. For the cluster galaxy population, our data yield a slope slightly steeper than of Zhang et al. (2011), while the contribution of the ICL steepens the slope such that at $M_{500} \approx 10^{14} \mathrm{M}_{\odot}$ the stars contribute $\sim 40 \%$ as much mass as the gas within $r_{500}$.

The slope in GZZ07, which corresponds to $b-1$, was $0.20 \pm 0.05$; here we report $0.26 \pm 0.03$. The normalization of the relation is also $8 \%$ higher than the one derived from the data in Vikhlinin et al. (2006), which is consistent with the offset in total mass presented in Figure 2 and 93 The uncertainty in the total masses due to both calibration and departures from hydrostatic equilibrium is our largest source of systematic uncertainty in the gas fractions. As discussed in section 5.4.2, the derived slope is robust to this uncertainty.

\subsubsection{Stellar Mass Fraction}

While a steep decrease in stellar baryon fraction with increasing cluster mass has been observed now by a number of groups (e.g., GZZ07; Giodini et al. 2009; Andreon 2010; Zhang et al. 2011; Lin et al. 2012), the most recent generation of simulations have difficulty reproducing this trend (e.g., Kravtsov et al. 2009; Puchwein et al. 2010; Young et al. 2011). The much weaker mass dependence found in these simulations, when coupled with their matching of the gas fraction versus halo mass relation, implies too weak a dependence of star formation efficiency on $M_{500}$. Additionally, Balogh et al. (2008) have argued on analytic grounds that slopes steeper that $\alpha \sim-0.3$ are difficult to reconcile with hierarchical structure formation in a $\Lambda \mathrm{CDM}$ cosmology — put simply, it is hard to form clusters with low stellar baryon fractions through the assembly of lower mass systems with higher stellar baryon fractions. In that study, the authors postulated that the GZZ07 results could potentially be reconciled with theoretical expectations if the velocity dispersion mass estimates were systematically biased for the lowest mass systems. The improved, independent masses in our current work directly address this concern.

We present in Fig. 7 updated data for stellar baryon fractions as a function of $M_{500}$. We overplot the best fit relation to the current data (solid line) compared with the best fit relation from GZZ07 (dotted). In GZZ07 we reported $\log f_{\star, 2 D} \propto(-0.64 \pm 0.13) \log \mathcal{M}_{500}$. Here we find that a best orthogonal distance regression fit to the full new set of data points yields $\log f_{\star, 2 D} \propto(-0.45 \pm$ $0.04) \log \mathcal{M}_{500}$, shallower than the previous value by 1.5 $\sigma$, but still significantly steeper than -0.3 .

One notable difference in the new stellar baryon fractions relative to GZZ07 is that the current data are less consistent with a simple power-law fit with low scatter. The few systems with $M<2 \times 10^{14}$ have stellar baryon fractions that on average lie below a simple extrapolation of the trend observed at higher mass. The ratio of stellar to gas mass however shows little scatter or departure from a pure power law over the full mass range. Any departure from a simple power-law must be driven by either lower true total baryon fractions or by residual bias in the $M_{500}$ determinations. We return to this point below in the context of total baryon fractions.

The overall normalization of the stellar baryon fraction relation (prior to deprojection) is similar to that in our previous work. While $\Upsilon_{\star}$ is $\sim 13 \%$ lower than in GZZ07 (4.2), the new $M_{500}$ values largely offset this change. The X-ray data used in this analysis enables us to avoid the conversion from $\sigma$ to $T_{x}$ to $M_{500}$ via scaling relations as in GZZ07, which is a clear improvement.

The application of a deprojection correction to estimate $M_{\star, 3 D}$ is therefore the most significant change relative to GZZ07. Because the BCG+ICL fraction decreases with $M_{500}$, deprojection slightly steepens the slope of the stellar baryon fraction relation $(0.48 \pm 0.04)$ in addition to decreasing the overall normalization by $\sim 20 \%$ (Figure 7). We list both the projected and deprojected stellar mass relations in Table [4. We focus upon $M_{\star, 3 D}$ in the next section when considering the total baryon content within $r_{500}$. The largest source of systematic uncertainty 


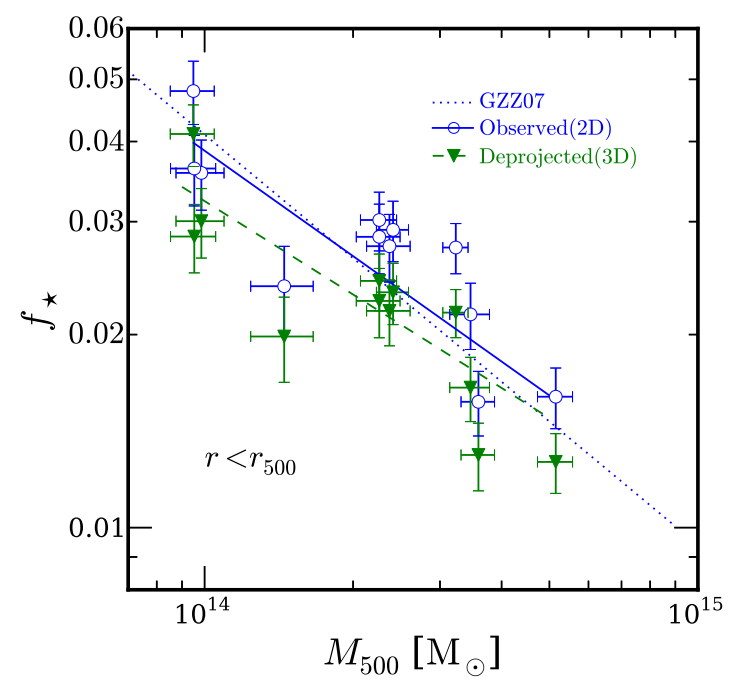

FIG. 7.- Stellar baryon fraction as a function of cluster mass for the systems in our sample. The blue open circles denote the stellar mass determined within a projected radius of $r_{500}$, and the solid line is the best fit to the dependence of observed stellar mass upon $M_{500}$ over the range covered by the current data. The dotted line is the best fit relation from GZZ07. Our current results confirm the trend in the stellar baryon fraction from our previous paper. The green filled triangles correspond to the same data points after applying a correction for projection effects to estimate the total stellar baryon content within a sphere of radius $r_{500}$ centered on the brightest cluster galaxy. This correction lowers the inferred stellar baryon fractions by $\sim 20$ percent, with the best fit in this case denoted by the dashed line.

in the stellar baryon fraction arises from uncertainty in the conversion from luminosity to stellar mass, which could change the normalization of the derived relation by up to $15 \%$ (see section 5.4.1). Uncertainty in the total mass is sub-dominant, having a minimal impact on the slope and normalization (see section 5.4.2).

\subsubsection{Total Baryon Mass Fraction}

The total baryon mass fractions within $r_{500}$ for each individual cluster are shown in the lower panel of Figure 8 for a WMAP7 cosmology. Above $2 \times 10^{14} \mathrm{M}_{\odot}$, the weighted mean baryon fraction for the current data (including only systems with both stellar and gas masses) is $f_{\text {bary }}=0.136 \pm 0.005$, which is $18 \%$ below the Universal value. For comparison, in GZZ07 we derived $f_{\text {bary }}=0.133 \pm 0.004$. In GZZ07 we found that the total baryon fraction was consistent with being independent of cluster mass. In contrast, the current data are consistent with a weak dependence of the baryon fraction upon cluster mass (Table 44). The derived power law slope is $0.16 \pm 0.04$. The updated stellar masses and deprojection correction have a minimal impact; the primary factor driving this change is the addition of X-ray data for use in deriving total and gas masses for individual systems. In contrast, the overall baryon fractions are higher, and the trend with mass weaker, than found by Leauthaud et al. (2012) using an extended halo occupation distribution approach (HOD; see their Figure 11). The similarity of our derived slope with the result of Giodini et al. (2009, $0.09 \pm 0.03)$ is likely coincidental. The stellar mass fraction relation in that work, which does not include the ICL, is high relative to most studies by an amount similar to our estimated additional ICL
TABLE 4

Derived $M_{j}-M_{500}$ Relations

\begin{tabular}{lcr}
\hline \hline Component & $a$ & $b$ \\
\hline$M_{\star, 2 D}$ & $3.9 \pm 0.2 \times 10^{-2}$ & $0.55 \pm 0.04$ \\
$M_{\star, 3 D}$ & $3.2 \pm 0.1 \times 10^{-2}$ & $0.52 \pm 0.04$ \\
$M_{\text {gas }}$ & $8.8 \pm 0.3 \times 10^{-2}$ & $1.26 \pm 0.03$ \\
$M_{\text {bary }}$ & $1.17 \pm 0.04 \times 10^{-1}$ & $1.16 \pm 0.04$ \\
\hline$M_{\star, 2 D} / M_{\text {gas }}$ & $4.75 \pm 0.02 \times 10^{-1}$ & $-0.82 \pm 0.05$ \\
$M_{\star, 3 D} / M_{\text {gas }}$ & $3.90 \pm 0.02 \times 10^{-1}$ & $-0.84 \pm 0.04$
\end{tabular}

Note. - Best fit parameters for the relation $M_{j}=$ $a\left(M_{500} / 10^{14} M_{\odot}\right)^{b}$, where $M_{j}$ is the mass contained in each baryonic component. $M_{\text {bary }}$ is derived using the deprojected stellar mass. The slope for the baryon fraction relations is equivalent to $1-b$. We also include the best fit parameters for the stellar-to-gas mass ratios as a function of $M_{500}$. The relation for the gas mass is derived including the clusters from Vikhlinin et al $(2006)$, while the other relations are derived using only clusters with both gas and stellar mass data.

contribution (Figure 10).

Systems with $M \lesssim 2 \times 10^{14} \mathrm{M}_{\odot}$ show evidence for larger scatter in $f_{\text {bary }}$ than do the higher mass systems, with total baryon fractions ranging from $60-90 \%$ of the Universal value. This large scatter, which is also discussed in Sanderson et al. (2013), is not however present in the ratio of stellar to gas mass? Even if the total baryon fraction within a halo varies from the mean expectation, the division of baryons between hot and cold components is relatively unaffected. Consequently, the observed scatter must be due to either intrinsic variations in the total baryon content within $r_{500}$ or remaining, unappreciated uncertainties in the derived $M_{500}$. While both the analyses in this work and in Sanderson et al. (2013) rely upon the same fundamental assumptions and include some of the same systems, the fact that both observe large scatter for low mass systems argues against a large bias in $M_{500}$ arising from details of the analysis method.

The existence of some systems at low mass with baryon fractions as high as those of massive clusters raises the possibility that there exists a flat upper envelope of baryon fractions across all masses. If so, then the observed weak trend could simply be due to smaller systems being increasingly susceptible to baryon loss and scattering downward from their initial baryon fractions. Conversely, it may be that there is an intrinsic trend of $f_{\text {bary }}$ with mass, but with increasing physical scatter with decreasing mass. We cannot currently distinguish between these alternatives.

\subsubsection{Impact of Cosmological Parameters}

To assess the impact of the choice of cosmological model, we repeat our entire analysis using the recently released cosmolgical parameters from Planck (Planck Collaboration et al. 2013) In Figure 9 we show the gas, stellar, and total baryon mass fractions derived using these parameters. The most striking difference relative to Fig. 8 is the reduced offset between the cluster total baryon mass fractions and the Universal value.

9 The baryon fractions presented in this paper are typically $\sim$ $10 \%$ higher than those in Sanderson et al. (2013) due to differences between the X-ray analyses, but the scatter is similar. 


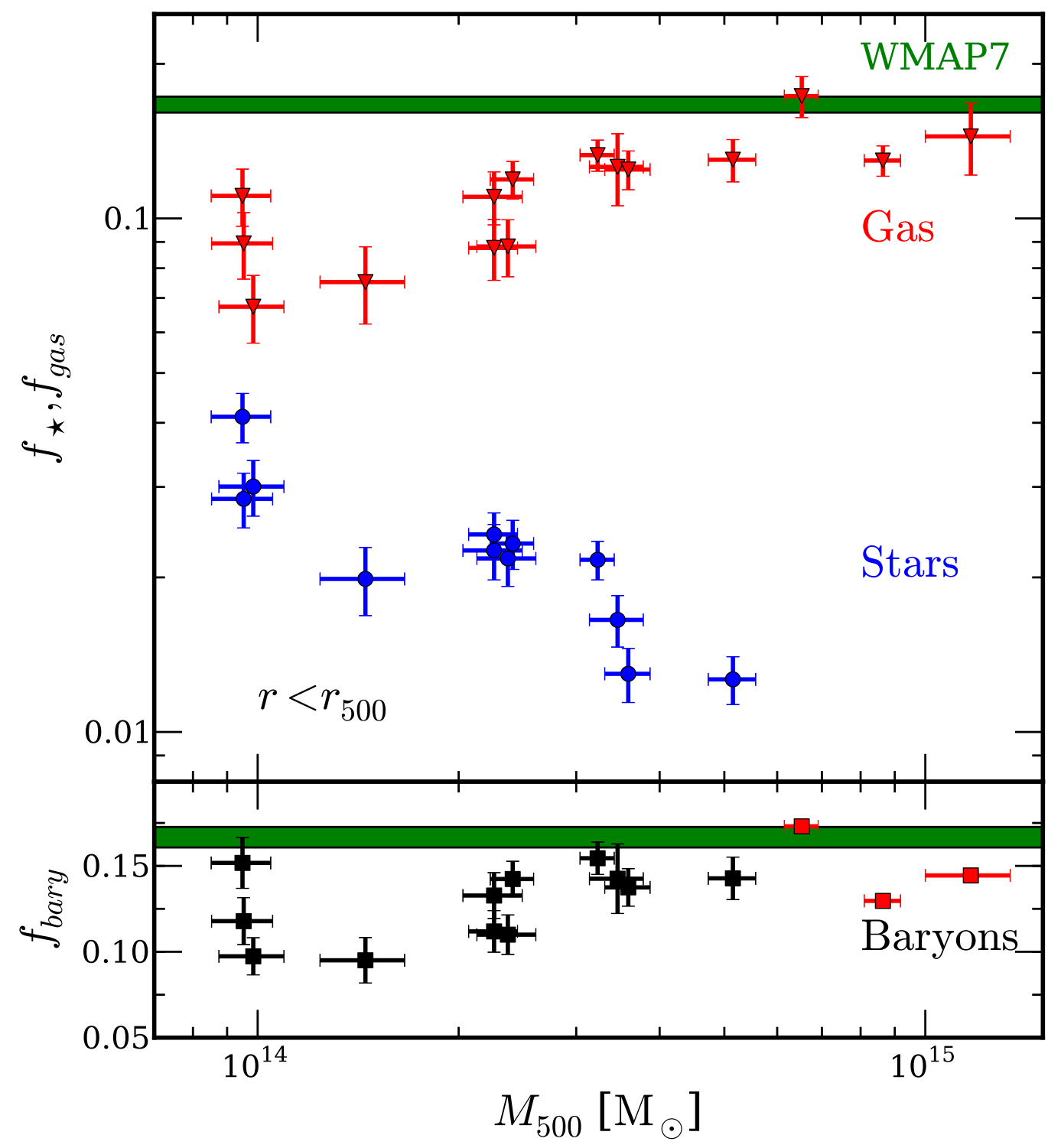

FIG. 8. - Stellar, gas, and total baryon fraction as a function of cluster mass for the systems in our sample. The stellar data (blue circles) include the deprojection correction, as discussed in the text. Our best fit to this stellar data is $f_{\star, 3 D} \propto M_{500}^{-0.48 \pm 0.04}$, confirming evidence for a steep slope in our previous work. The gas fractions (red triangles) are plotted for all systems including the massive clusters from Vikhlinin et al. (2006) for which we lack stellar fractions. The opposing trends for gas and stellar baryon fractions imply strongly decreasing star formation efficiency with increasing cluster mass. The total baryon fractions in the lower panel are plotted as black squares for all systems with stellar and gas data. We also include as red squares the gas fractions for the most massive clusters that lack stellar data; these are formally lower limits on the total baryon fractions, with stellar baryon fractions expected to be at the level of $f_{\star} \sim 0.01-0.02$. The total baryon fractions are consistent with a weak, but statistically significant dependence of the total baryon fraction upon $M_{500}\left(f_{\text {bary }} \propto M_{500}^{0.16 \pm 0.04}\right)$ over the mass range where we have both stellar and gas measurements. This trend is driven primarily by the lower mass systems, for which we also see evidence of large scatter in the total baryon fractions. The weighted mean baryon fraction for the current data at $M>2 \times 10^{14} \mathrm{M}_{\odot}, f_{\text {bary }}=0.136 \pm 0.005$, is $18 \%$ below the Universal value.

Above $2 \times 10^{14} \mathrm{M}_{\odot}$, the weighted mean baryon fraction is $f_{\text {bary }}=0.144 \pm 0.005$, which is only $7 \%$ below the Universal value from Planck. Meanwhile, the change in parameters has minimal impact on either the derived slopes (see Appendix), or on the scatter at lower mass, with total baryon fractions ranging from $65-100 \%$ of the Universal value from Planck.

\subsection{Potential Systematic Biases}

In the preceeding sections we showed that the BCG+ICL contribute appreciably to the total stellar luminosity, especially for low mass systems, confirmed that the stellar and gas fractions are strong, inverse functions of $M_{500}$, and demonstrated that the gas-to-stellar baryon ratio is tightly correlated with $M_{500}$. We also find that the total baryon fraction is $\sim 17 \%$ (7\%) below the Universal values for systems with $M_{500} \gtrsim 2 \times 10^{14} \mathrm{M}_{\odot}$ when using cosmological parameters from the WMAP7 (Planck) analysis, with a weak but statistically significant dependence upon $M_{500}$. We now assess the amplitudes of biases that may affect the stellar, gas, and total mass determinations within $r_{500}$, and consider the extent to which they may alter our conclusions. 


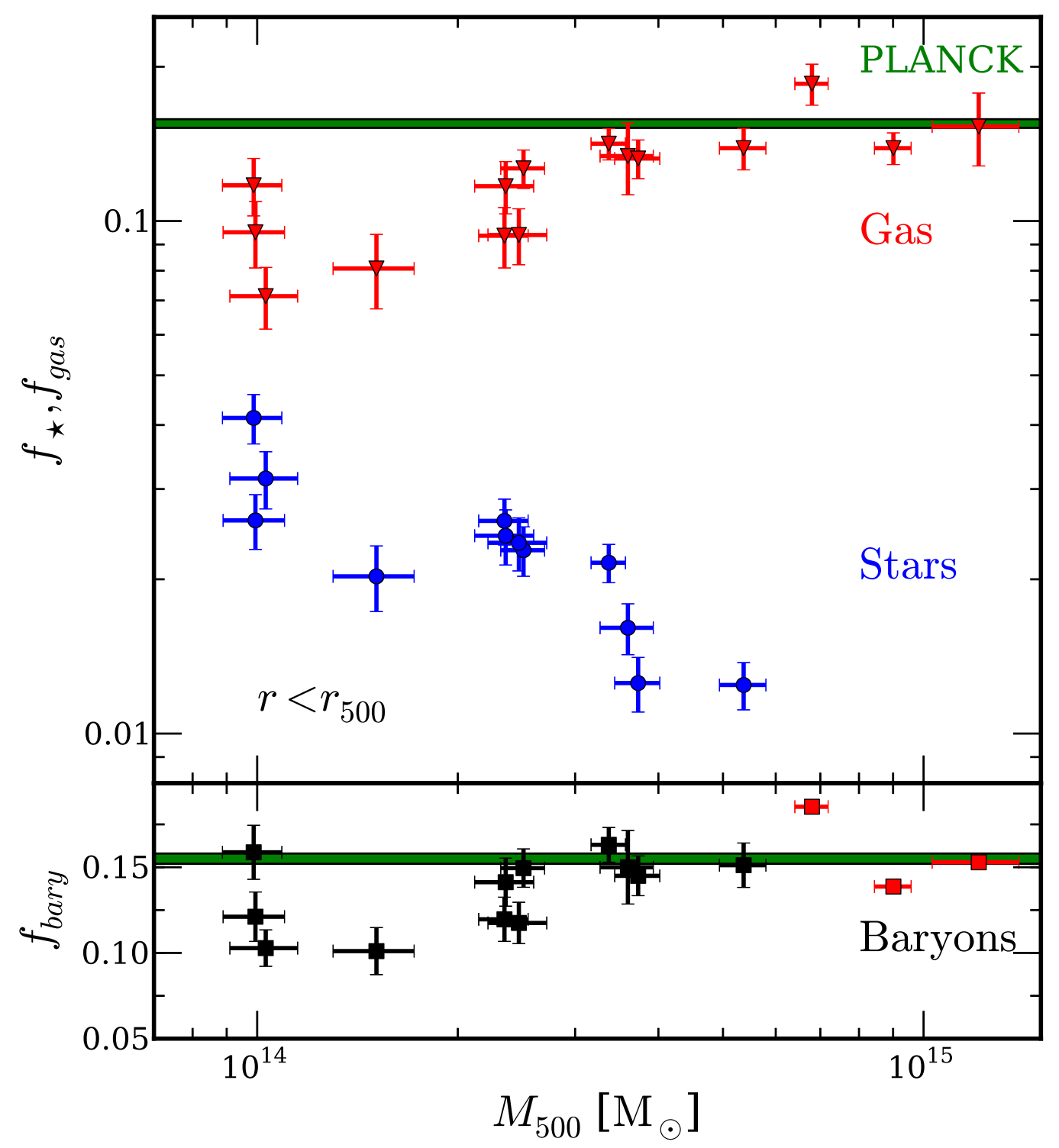

FIG. 9.- Same as Fig. 8 but using the Planck cosmological parameters from (Planck Collaboration et al. 2013). The best fit slopes to the stellar and gas relations, which are provided in the Appendix, are minimally affected by the change in cosmology. The derived total baryon fractions however are closer to the Universal value when using the Planck cosmological parameters. The weighted mean baryon fraction for the current data at $M>2 \times 10^{14} \mathrm{M}_{\odot}, f_{\text {bary }}=0.144 \pm 0.005$, is only $7 \%$ below the Universal value.

\subsubsection{Stellar Mass}

For the stellar masses, the two stages in which biases might arise are determination of the total luminosity and the conversion to stellar mass. As noted earlier, uncertainty in the faint end slope of the galaxy luminosity function induces a $12 \%$ uncertainty in the integrated luminosity of the galaxy population, which corresponds to a $5-8 \%$ uncertainty in the total luminosity including the BCG and ICL. The luminosity of the intracluster light can also be underestimated if there is a more extended component at very low surface brightness levels that is not co-centric with the BCG. We see no evidence at higher surface brightness levels for such a component, but include the possibility here for completeness.

A more immediate concern, as mentioned in 4.2 is the conversion of luminosity to stellar mass. There are several approaches that one can take for this conversion. One approach is to derive $\Upsilon_{\star}$ based upon stellar popu- lation models using an assumed spectral energy distribution. This approach can either be done using singleband photometry and a mean conversion for the ensemble galaxy population (Lin et al. 2003; Giodini et al. 2009; Zhang et al. 2011; Laganá et al. 2011), or preferrably using multiband photometry to fit for stellar masses for each individual cluster galaxy (Leauthaud et al. 2012). Deriving an independent $\Upsilon_{\star}$ for each galaxy has the advantage of making the integrated results insensitive to variations in the passive galaxy fraction from cluster to cluster.

All $\Upsilon_{\star}$ estimates based upon stellar population models share a common weakness, however, which is that they are only as accurate as the underlying assumptions. Specifically, the derived $\Upsilon_{\star}$ is strongly sensitive to assumptions about the shape of the initial mass function and to systematic uncertainties in the stellar population models themselves (see e.g., Conrov et al. 2009, for a de- 


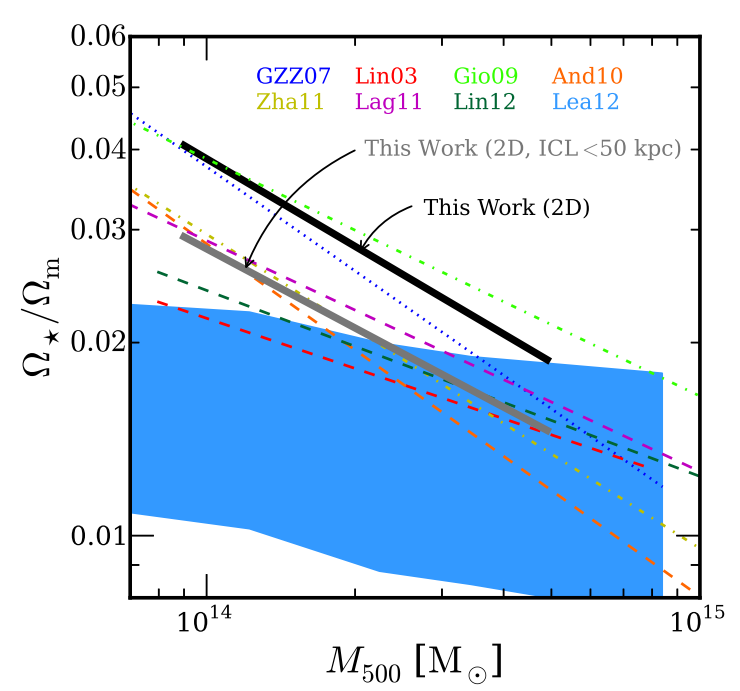

FIG. 10.- Comparison of the stellar baryon fraction relations in the literature with our observed relations, which illustrates that our $\sim 25 \%$ higher normalization is due to the significant contribution of ICL beyond $50 \mathrm{kpc}$, which is generally not included in other studies. The increasing importance of ICL in low mass systems also acts to steepen the slope of the relation. We include published results from Lin et al. (2003, Lin03), Giodini et al. (2009, Gio09), Andreon (2010, And10), Zhang et al. (2011, Zha11), Laganá et al. (2011, Lag11), Lin et al. (2012, Lin12), and Leauthaud et al. (2012, Lea12). The Andreon (2010) relation is derived within $r_{200}$, while all others are within $r_{500}$. The Lin et al. (2003), GZZ07, and Andreon (2010) mass-to-light ratios are based upon dynamical measurements, while the rest are shown for a Salpeter IMF. In the case of Lin et al. (2012), we have scaled the stellar mass up by a factor of 1.34 to convert from Kroupa to Salpeter IMF, while for Leauthaud et al. (2012) we have used the shaded region corresponding to a Salpeter IMF from their Figure 5. For GZZ07 we have changed the mass-to-light ratio to match that used in this paper. The thick, solid black line is our observed relation within $r_{500}$ If we approximately remove the contribution of the ICL beyond 50 $\mathrm{kpc}$, we recover a relation similar in both slope and normalization to most other published studies within $r_{500}$ ("50 kpc" case; thick, solid grey line). If we apply a deprojection correction to our observed relation, the resulting normalization is similar to that of the relation for the "50 kpc" case - the contribution from the ICL at larger radii and the amplitude of the deprojection correction are roughly equal and opposite. Both relations are inconsistent with the Leauthaud et al. (2012) HOD results, particularly at low mass.

tailed description of these uncertainties), which can result in a significant mis-estimation of the stellar baryon fraction. Moreover, recent results from Cappellari et al. (2012) argue that the initial mass function is not universal, but rather is dependent upon galaxy mass - implying a different conversion than under the assumption of a universal IMF.

An alternative approach is to estimate $\Upsilon_{\star}$ using dynamical measurements of the central mass-to-light ratio for nearby galaxies. The dynamical approach, while less sensitive to the IMF, is not entirely free of assumptions. The measured dynamical mass includes both stellar and dark matter contributions, providing a direct observed upper limit on $M_{\star}$. One must then estimate the fractional contribution of dark matter to the dynamical mass in the central regions of galaxies in order to estimate the stellar mass-to-light ratio. The standard technique for estimating the dark matter fraction involves comparing the total dynamical mass with the stellar mass one infers from stellar population models. Using this technique,
Cappellari et al. (2006) found that the dark matter contribution can be up to $30 \%$ in individual systems for a Kroupa IMF, but it is generally lower for slow rotators, like the massive galaxies that contribute most to the total stellar mass.

A number of recent studies find evidence for IMFs as steep as, or steeper than, Salpeter in massive elliptical galaxies (Conrov \& van Dokkum 2012; van Dokkum \& Conroy 2012; Cappellari et al. 2012), and other stellar systems (Zaritsky et al.|2012). The average contribution of $15 \%$ used in this paper is chosen as the midpoint of values spanned by the range of input assumptions. The associated systematic uncertainty is therefore at most $15 \%$ even considering the impact of the IMF. This remaining uncertainty changes the slope of the relation between total baryon mass and $M_{500}$ by $<1 \sigma$. Only if the IMF of cluster galaxies were correlated with $M_{500}$ could one achieve a larger change in slope. Future results from the ATLAS ${ }^{3 \mathrm{D}}$ survey (Cappellari et al. 2011) should further reduce this systematic uncertainty.

The impact of the star-forming population upon the mean $\Upsilon_{\star}$, which we have previously ignored, is a more subtle issue. There are several factors to consider. First, if the star-forming fraction depends upon $M_{500}$ then this will induce a bias that scales with cluster mass, as noted by Leauthaud et al. (2012). Recent studies yield somewhat contradictory results for this dependence. Both Finn et al. (2008) and Balogh \& McGee (2010) find that the fraction of star-forming galaxies is nearly independent of mass from group to cluster scales, while Weinmann et al. (2006) and Wetzel et al. (2012) conclude that the passive fraction does increase with mass. For the mass range under consideration in this paper, $M>10^{14} \mathrm{M}_{\odot}$, the change is however minimal. For example, in Wetzel et al. (2012) the passive fraction increases by $\sim 5 \%$ between $10^{14} \mathrm{M}_{\odot}$ and $10^{15} \mathrm{M}_{\odot}$ for massive cluster galaxies $\left(M_{\text {stellar }} \gtrsim 5 \times 10^{11} \mathrm{M}_{\odot}\right)$, with a $<10 \%$ increase even for the lowest mass clusters in that study. If we assume the extreme, limiting case of $\Upsilon_{\star}=0$ for star-forming galaxies, then a $10 \%$ decrease in the passive fraction changes the inferred $\Upsilon_{\star}$ by only $4 \%$ if the ICL contribution is still assumed to be purely passive.

Second, the presence of star-forming galaxies in the cluster means that we will systematically overestimate $\Upsilon_{\star}$ because we are assuming a purely quiescent population. To gauge the amplitude of this effect for our data we use the formalism described in Lin et al. (2003). As inputs to this calculation we take the true passive fraction among cluster galaxies to be $\sim 75 \%$ (Balogh \& McGee 2010) and consider the BCG+ICL as an additional purely passive stellar population, using the BCG+ICL fractions in Fig. 5. We explore the impact of three values of $\Upsilon_{\star, I}$ for the star-forming population: $0.5,1$, and 2 . If we weight by the relative contributions of the passive and star-forming stellar populations then the composite $\Upsilon_{\star, I}$ decreases by $14 \%, 12 \%$, and $7 \%$, respectively. Recently quenched galaxies will also have lower $\Upsilon_{\star}$ than galaxies whose star formation ended earlier. The overall impact of this population is expected to be small given the rarity of post-starburst galaxies in local clusters (e. g. Couch et al. 2001; Poggianti et al. 2004).

Third, the $\Upsilon_{\star}$ values in Cappellari et al. (2006) are 
derived for a sample of local galaxies. The galaxy clusters in our sample lie at $z \simeq 0.1$, and evolution since $z \sim 0.1$ acts to increase $\Upsilon_{\star}$ with time. In our current analysis we make the approximation that this evolution is passive, as in GZZ07. Any residual systematic related to this evolutionary correction will be minor and subdominant to other sources of systematic and statistical uncertainty.

Another concern is that in our analysis we have treated the BCG and ICL as having the same $\Upsilon_{\star}$ as the luminosity-weighted value for the cluster population. For the BCG, this approach likely underestimates $\Upsilon_{\star}$. A BCG with a velocity dispersion of $\sim 300 \mathrm{~km} \mathrm{~s}^{-1}$ is expected to have $\Upsilon_{\star, I} \simeq 4.8$ based upon Cappellari et al. (2006). In GZZ05 we found that the BCG typically contributes $\sim 10-20 \%$ of the total light in the BCG+ICL, implying a net $\Upsilon_{\star}$ for the BCG+ICL $8-16 \%$ higher than the value assumed here. Given that the fraction of light in the BCG+ICL is $<50 \%$ (Figure 5 ), this corresponds to a potential bias of $<10 \%$ for the total cluster stellar masses.

As for the ICL, which dominates the luminosity of the BCG $+\mathrm{ICL}$, using the same $\Upsilon_{\star}$ as for the galaxy population is a reasonable approximation if ICL predominantly originates from tidal stripping and tidal disruption of cluster galaxies, consistent with theoretical predictions (Purcell et al. 2007; Conrov et al. 2007; Behroozi et al. 2012; Watson et al. 2012; Contini et al. 2013). Observational constraints on ICL color gradients support this picture: the color of the ICL lies within the range found for the cluster galaxy population (Zibetti et al.|2005, De Maio et al., in prep). The $\Upsilon_{\star}$ of the ICL would need to differ from the luminosity-weighted $\Upsilon_{\star}$ of the cluster galaxy population by more than $30 \%$ for this to be the dominant systematic uncertainty, an implausible level given current observational constraints.

Finally, one can ask whether there is any systematic uncertainty introduced by our methodology. As discussed in 4 , our approach has been to sum up the total luminosity, apply a background correction, and then apply a single $\Upsilon_{\star}$ weighted by a typical cluster luminosity function. An alternate approach is to compute the stellar mass of each galaxy individually prior to the background subtraction, assuming that it lies at the cluster redshift.

To assess the impact of the choice of methodology, we have repeated our analysis with this alternate approach. For both the cluster and background regions, we assign each galaxy an $\Upsilon_{\star}$ according to its magnitude using the Cappellari et al. (2006) relation and assuming that it lies at the cluster redshift, and then subtract off the background contribution. As before, we apply a completeness correction to account for the contribution of cluster galaxies fainter than the magnitude limit of our data. For the $\mathrm{BCG}+\mathrm{ICL}$, we use $\Upsilon_{\star}=2.65$ as in or main analysis. We again assume that all galaxies are quiescent, and refer the reader to the discussion above on the impact of star-forming galaxies upon the integrated stellar mass. We find that this alternate approach yields total stellar masses that are on average $15 \%$ lower, corresponding to a systematic uncertainty comparable in amplitude to the uncertainty associated with the dark matter contribution in the Cappellari et al. (2006) relation. The slope of the stellar mass fraction relation meanwhile steepens by $1.5 \sigma$. For the deprojected case it changes from $f_{\star, 3 D} \propto M_{500}^{-0.48}$ to $f_{\star, 3 D} \propto M_{500}^{-0.54}$.

Considering all of the above factors, the largest systematic uncertainties in our conversion to stellar mass are clearly defined and impact our results at a level of $15 \%$. We therefore consider this approach a robust alternative to the use of stellar population models, which have much larger systematic uncertainty arising from the IMF. For the total baryon fraction this corresponds to a maximum systematic uncertainty of $<5 \%$, given that $M_{\star} / M_{g a s}<0.5$ for all systems in our study.

A final consideration is potential bias arising from observing the projected stellar mass within $r_{500}$ and applying a deprojection correction to estimate the true stellar mass enclosed within a sphere of radius $r_{500}$. For this correction we assume an NFW profile with a concentration $c=2.9$ for the galaxy population (Lin et al. 2004), and apply no correction to the more compact BCG+ICL component. As discussed in \$5.3.2 this concentration implies that $71 \%$ of the stellar mass associated with the galaxy population within a projected radius of $r_{500}$ lies within a sphere of radius $r_{500}$. There are several other estimates in the literature where the concentration is measured in a similar fashion to Lin et al. (2004). Carlberg et al. (1997) found $c \simeq 3.7$ for CNOC clusters, while Budzynski et al. (2012) find $c \simeq 2.6$ using a sample of over 50,000 clusters and groups from the Sloan Digital Sky Survey. Notably, Budzynski et al. (2012) find that this concentration is approximately independent of cluster mass over the mass range covered by our study, indicating that any systematic uncertainty associated with the mass dependence of the concentration is subdominant to the uncertainty in the overall normalization. The Carlberg et al. (1997) and Budzynski et al. (2012) concentration values would give fractions of $73 \%$ and $69 \%$ of the projected stellar mass lying within a sphere of radius $r_{500}$, respectively. Thus, in the mean, the choice of concentration value can induce a few percent bias in the stellar mass associated with the galaxy population. The impact on the total stellar mass should thus be $\sim 1-2 \%$, depending on the contribution of the BCG+ICL for a given system.

\subsubsection{Gas Mass and $M_{500}$}

As noted in 5.3 .1 , our gas fractions are on average $8 \%$ higher than those derived by Vikhlinin et al. (2006) for the same systems. The gas fractions in general are offset by a similar amount relative to the relation from Vikhlinin et al. (2009) at high mass $\left(M_{500} \gtrsim 3 \times 10^{14}\right.$ $\left.\mathrm{M}_{\odot}\right)$, though they span a similar range at lower mass as both the Vikhlinin et al. (2009) and Sun et al. (2009) data. The primary origin of this offset is expected be the $8 \%$ smaller $M_{500}$ values we derive from the $X M M$ data. This remaining systematic uncertainty in $M_{500}$ is one of the two dominant systematic uncertainties in the current analysis (the other being uncertainty in $\Upsilon_{\star}$ ). As discussed in 93.4 any residual systematic error in $M_{500}$ is to first order expected to be a constant fractional error independent of mass. It therefore should only impact the overall normalization of the gas fractions.

A related concern is whether there exists any systematic bias endemic in gas-based $M_{500}$ measurements due to departures from hydrostatic equilibrium. The amplitude and direction of this systematic remains a topic of significant ongoing research activity. While simulations 
by Lau et al. (2013) indicated that the bias should be at the few percent level, recent studies comparing weak lensing and X-ray mass determinations within $r_{500}$ do not yet unambiguously resolve this issue. Zhang et al. (2010) find minimal offset for their full cluster ensemble, but a dichotomy between disturbed and relaxed clusters, with the X-ray masses exceeding the lensing masses by $6 \%$ for disturbed systems and being $9 \%$ low for relaxed systems. (Mahdavi et al. 2013) meanwhile find that for their full sample the X-ray masses are low by $12 \%$ relative to lensing, but with the cool-core systems having larger relative X-ray masses.

We consider this potential bias associated with the hydrostatic equilibrium approximation to be an outstanding issue that may impact our $M_{500}$ determinations by up to $\sim 15 \%$. If, for example, our derived $M_{500}$ (and hence $\left.r_{500}\right)$ are too low then $M_{\text {gas }}$ and $M_{\star}$ will also be underestimated due to their dependence on $r_{500}$. For a $15 \%$ increase in $M_{500}, f_{\text {gas }}$ and $f_{\star}$ should decrease by $\sim 8 \%$ and $\sim 3 \%$, respectively (c.f. Sanderson et al. 2013). The slopes of the gas and stellar fraction relations are negligibly affected by such bias, regardless of whether the masses are biased high or low.

One final potential concern is that our stellar mass measurements are derived in an aperture centered on the BCG, while the gas and total masses are derived within an equivalent aperture about the centroid of the X-ray emission. In practice, this difference in centering has a minimal impact on our results. As can be seen in Figure 1. the offsets between the BCG and X-ray emission are generally not large. For all but three clusters this offset is less than $20 \mathrm{kpc}$, while the maximum offset is $75 \mathrm{kpc}$ for Abell S0296. As a consistency check, for the systems with the largest offsets we recalculate the total baryon fractions with common centers, finding that they change by $<1 \%$ in all cases.

\subsection{Comparison with Other Studies}

It is useful at this point to compare the stellar baryon fractions derived in this work with the literature to assess the level of consistency among different studies. In particular, we consider results from Lin et al. (2003), Giodini et al. (2009), Andreon (2010), Laganá et al. (2011), Zhang et al. (2011), Lin et al. (2012), and Leauthaud et al. (2012). These studies use a range of techniques to quantify the stellar baryon fraction, each with different associated systematics, and thus the ensemble provides an indication of the overall uncertainty in cluster stellar baryon fractions. To enable as fair a comparison as possible, it is worthwhile to consider the key ways in which the methods differ.

There are three key issues. First, this paper and GZZ07 are the only studies that directly consider the contribution of the ICL to the stellar baryon content. The other studies do include the BCG, either directly (Lin et al. 2003; Giodini et al. 2009; Andreon 2010; Laganá et al. 2011; Zhang et al. 2011; Lin et al. 2012), or by virtue of an extended HOD formalism (Leauthaud et al. 2012). If we make the rough approximation that the BCG magnitudes in these papers correspond to the total light enclosed within the central 50 $\mathrm{kpc}$, then the BCG luminosities will include $\sim 40 \%$ of the combined BCG+ICL luminosity (Gonzalez et al. 2005). For direct comparison with previous studies we calculate
TABLE 5

Best Fit Parameters For Baryon Fraction Relations

\begin{tabular}{llc}
\hline \hline \multicolumn{1}{c}{ Source } & Slope $(b-1)$ & comments \\
& & \\
\hline Lin et al. (2003) & $-0.26 \pm 0.09$ & \\
Lin et al. (2012) & $-0.29 \pm 0.04$ & \\
Laganá et al. (2011) & $-0.36 \pm 0.17$ & \\
Giodini et al. (2009) & $-0.37 \pm 0.04$ & No ICL at $r>50 \mathrm{kpc}(2 \mathrm{D})$ \\
This paper & $-0.38 \pm 0.05$ & Including full ICL (2D) \\
This paper & $-0.45 \pm 0.04$ & Including full ICL (3D) \\
This paper & $-0.48 \pm 0.04$ & \\
Zhang et al. (2011) & $-0.49 \pm 0.09$ & Within $r_{200}$ \\
Andreon (2010) & $-0.55 \pm 0.09$ & Including full ICL (2D) \\
GZZ07 & $-0.64 \pm 0.13$ & \\
\end{tabular}

Note. - Slopes are sorted in order of decreasing value. All values are computed for $r_{500}$ except for Andreon (2010).

the inferred stellar baryon fraction that we would obtain if we were to use a similar approach. Excluding $60 \%$ of the BCG+ICL luminosity in this fashion yields a shallower stellar baryon relation, $f_{\star, 2 D} \propto M_{500}^{-0.38 \pm 0.05}$. This slope lies near the median of values derived in recent literature within $r_{500}$ which range from -0.26 to -0.49 (see Table 5 and Figure 101).

Second, Andreon (2010) derives the stellar baryon fraction within $r_{200}$ rather than $r_{500}$, and derives a relation between stellar mass and $\mathrm{M}_{200}$. This choice of aperture clearly provides a more complete census of the stellar baryon content. It also mitigates the impact of not including the ICL, leading to a steeper slope that is consistent with the slope we derive when including the ICL. Unfortunately, use of this radius means that the normalization is not directly comparable with our studies and others, which select $r_{500}$ as a radius within which the gas content can also be accurately measured. We still include the Andreon (2010) relation in Fig. 10, but emphasize this key difference relative to other studies.

Third, each of the studies listed uses a different $\Upsilon_{\star}$ (or in the case of Leauthaud et al. (2012) a distribution of $\Upsilon_{\star}$ values) and is based upon data sets at different redshifts. As discussed above, the conversion from luminosity to stellar mass is easily the dominant source of systematic uncertainty.

We make a best attempt in Figure 10 to compare the various studies on relatively equal footing. In this Figure we show the literature results for a Salpeter (1955) IMF for those studies based upon stellar population models. In the case of Lin et al. (2012), which is based upon a Kroupa (2001) IMF, we use the prescription in their paper (multiplying by a factor of 1.34) to convert to a Salpeter IMF. Those studies that use dynamical $\Upsilon_{\star}$ values are plotted as published (Lin et al. 2003; Gonzalez et al. 2007; Andreon 2010). We also plot the band derived from the extended HOD formalism in Figure 5 of Leauthaud et al. (2012) for a Salpeter IMF, where the width of the band reflects associated systematic uncertainties. We note that the stellar masses used by Giodini et al. (2009) have subsequently been found to be biased high (Leauthaud et al. 2012; Giodini et al. 2012), contributing to the relatively high normalization of that relation.

While there are several relations that are outliers relative to the ensemble, we recover a normalization and 
slope that approximate the median of the literature values if we exclude the fraction of the ICL that is expected to be missed by these studies. Our total stellar baryon fraction relation including the ICL remains similar in amplitude to the relation in GZZ07. When we add a deprojection correction to the stellar mass, which was not done in GZZ07, the resultant normalization is similar to that of the "50 kpc" relation plotted in Figure 10, which excludes the contribution of the ICL beyond $50 \mathrm{kpc}$ from the BCG - the ICL contribution and deprojection correction are roughly equal and opposite in amplitude.

It is clear that the Leauthaud et al. (2012) extended HOD results are significantly lower than direct $f_{\star}$ determinations, particularly at low mass where the inclusion of the ICL further exacerbates this tension (though at even lower mass scales they are consistent with the direct measurements in Leauthaud et al. (2012)). This fact was discussed in Leauthaud et al. (2012) in the context of a comparison with GZZ07 and Giodini et al. (2009). Leauthaud et al. (2012) suggested that the offset between their work and the GZZ07 results is due to use of an excessively large $\Upsilon_{\star}$ in GZZ07. In this paper we have carefully recalculated the dynamical $\Upsilon_{\star}$ values, reconsidering all assumptions to assess the level of remaining systematic uncertainties. While the value of $\Upsilon_{\star}$ used in this paper is $26 \%$ lower than in GZZ07 the offset with Leauthaud et al. (2012) remains despite our efforts to place all studies on equal footing. The reason for this tension is unclear, but certainly warrants further investigation.

\section{CONCLUSIONS}

The central goal of this paper is to extend the main results from Gonzalez et al. (2007) using a sample for which we possess total, gas, and stellar mass measurements for each cluster. The combination of these measurements for individual systems addresses the two main weaknesses of our previous analysis: the lack of robust cluster mass estimates used to derive the stellar mass scaling relation and the use of disjoint data sets for the gas and stellar mass measurements. As a result, the total baryon fractions in GZZ07 were based upon scaling relations rather than derived for individual systems. Here we present an analysis of the total baryon fraction within $r_{500}$ for a sample of clusters with complete data on the intracluster medium and stellar component. These data also enable a direct comparison of the partitioning of baryons between gas and stars for individual systems and for stars between galaxies and the brightest cluster galaxy plus intracluster light $(\mathrm{BCG}+\mathrm{ICL})$ component. Our central findings are:

1. We confirm the trend of steeply decreasing stellar mass fraction with cluster mass seen in GZZ07, which has now been verified by multiple groups for the cluster galaxy population without inclusion of intracluster light $(-0.3 \gtrsim \alpha \gtrsim-0.55$, Table 5. Giodini et al. 2009; Andreon 2010; Zhang et al. 2011; Lin et al. 2012). Our best fit to the current data has a scaling $f_{\star, 2 D} \propto M_{500}^{-0.45 \pm 0.04}$, including the BCG+ICL; if we exclude the fraction of the ICL that we expect is typically missed by other studies, then we recover $f_{\star, 2 D} \propto M_{500}^{-0.38 \pm 0.05}$ with a $26 \%$ lower normalization at $10^{14} \mathrm{M}_{\odot}$ (Figure 10).
The current data are less consistent with a simple power-law fit with low scatter than our previous data. In particular, the stellar baryon fractions at $M<2 \times 10^{14} \mathrm{M}_{\odot}$ on average are lower than expected from a simple extrapolation of the trend observed at higher mass. The limited existing data however make any firm conclusions premature. We also recover a scaling relation for the hot gas, $f_{\text {gas }} \propto M_{500}^{0.26 \pm 0.03}$, with a slope similar to that of the relation used in our previous work and consistent with other determinations in the literature.

2. The combination of brightest cluster galaxy plus intracluster light is confirmed to contain an decreasing fraction of the total luminosity within $r_{500}$ with increasing cluster mass. For systems with $M_{500} \approx$ $10^{14} \mathrm{M}_{\odot}$, the BCG+ICL together typically contribute $40-50 \%$ of the total luminosity within $r_{500}$. As discussed in GZZ07, the decrease in the importance of the BCG+ICL, and particularly the ICL, with increasing cluster mass is consistent with less efficient tidal stripping and disruption of galaxies in the more massive systems. We do note that one of the $M_{500} \approx 10^{14} \mathrm{M}_{\odot}$ clusters from Sanderson et al. (2013) has a lower BCG+ICL luminosity fraction that is similar to more massive systems. This system suggests that there may be significant scatter in the BCG+ICL luminosity fraction at this mass scale, but a larger sample will be required to clarify this point.

3. The partitioning of baryons between stars and gas within $r_{500}$, a measure of the integrated efficiency with which baryons are converted to stars, is strongly correlated with $M_{500}$. Our results for the galaxy population, excluding ICL beyond 50 kpc from the BCG, yield a relation, $M_{\star, 2 D} / M_{\text {gas }} \propto$ $M_{500}^{-0.79 \pm 0.05}$. This power law slope is $2.5 \sigma$ steeper than that found by Zhang et al. (2011). While differences in the X-ray analyses may contribute, we cannot identify any single reason for this $2.5 \sigma$ difference. Inclusion of the entire contribution from the ICL, which constitutes a greater fraction of the stellar mass at low cluster mass, and applying a deprojection correction both further steepen this slope, yields $M_{\star, 3 D} / M_{\text {gas }} \propto M_{500}^{-0.82 \pm 0.05}$. If the observed trend continues to lower mass, then the stellar contribution may be $>50 \%$ on mass scales $M_{500} \lesssim 3 \times 10^{13} \mathrm{M}_{\odot}$.

4. The derived relations for $M_{\star, 2 D} / M_{\text {gas }}$ and $M_{\star, 3 D} / M_{g a s}$ versus $M_{500}$ demonstrate a stronger dependence of star formation efficiency upon cluster mass than the most recent generation of numerical simulations are able to reproduce (e.g., Puchwein et al. 2010; Young et al. 2011). For the $M_{\star, 3 d} / M_{\text {gas }}-M_{500}$ relation we derive an intrinsic scatter of only $12 \%$. This low scatter in the stellarto-gas mass relation implies that the star formation efficiency at a fixed cluster mass scale is quite uniform. It further argues that in the $f_{*}-M_{500}$ relation any departure from a power law at low mass must be attributed to either the $M_{500}$ determina- 
tions or changes in the total baryon content rather than to variations in the star formation efficiency.

5. For systems with $M_{500} \geq 2 \times 10^{14} \mathrm{M}_{\odot}$, the average total baryon fraction is $f_{\text {bary }}=0.136 \pm 0.005$, $18 \%$ below the Universal value for the WMAP7 cosmology. Use of the Planck cosmological parameters raises the derived total baryon fraction to $f_{\text {bary }}=0.144 \pm 0.005$ for these systems, which at only $7 \%$ below the Universal baryon fraction is consistent with this Universal value to within the current systematic uncertainties For this Planck cosmology there are essentially no missing baryons within $r_{500}$ for massive galaxy clusters. In GZZ07, we concluded that the total baryon fraction was consistent with being independent of halo mass on group to cluster scales. With the present, improved data, we now see a modest increase with mass of the total baryon fraction, $f_{\text {bary }} \propto M_{500}^{0.16 \pm 0.04}$. Below $2 \times 10^{14} \mathrm{M}_{\odot}$, our results suggest an increasing physical spread in the total baryon fraction among systems (see also Sanderson et al. 2013), with fractions ranging from $60-90 \%(65-100 \%)$ of the WMAP7 (Planck) Universal value. Such a variation could arise in a number of ways, including variance in the initial conditions or redistribution of baryons to beyond $r_{500}$. However, the relative tightness of the $M_{\star} / M_{\text {gas }}$ relation, even at these lower halo masses, suggests that this scatter must arise in a fashion that affects both the stellar and gaseous components equally, arguing against latetime hydrodynamic redistribution (e.g., via feedback) of some baryons beyond $r_{500}$.

6. Uncertainties associated with the conversion of luminosity to stellar mass, and with the X-ray derived $M_{500}$, are the most important sources of systematic uncertainty for the derived stellar baryon fractions. The assorted systematics associated with the conversion to stellar mass are present at the level of $\sim 15 \%$. We advocate use of dynamical estimates for $\Upsilon_{\star}$ due to the minimal required assumptions, and advise caution in interpreting results that rely on $\Upsilon_{\star}$ values derived using stellar population models, for which the choice of IMF can change the inferred stellar mass by nearly a factor of two (e.g., Leauthaud et al. 2012). Additionally, the recent Cappellari et al. (2011, 2013) results suggest variation in the shape of the initial mass function, and hence $\Upsilon_{\star}$, with galaxy mass. If correct, then current analyses that presume a single
IMF at all mass scales will yield inherently biased estimates of $\Upsilon_{\star}$.

For $M_{500}$ both instrumental calibration and departures from hydrostatic equilibrium are potential sources of bias. We note that our derived $M_{500}$ are systematically lower than those from Vikhlinin et al. (2006) by $8 \%$, providing an approximate estimate of the magnitude of calibration uncertainty. As discussed in the text, literature comparisons also indicate that weak lensing $M_{500}$ determinations may be up to $15 \%$ higher than X-ray values. The net impact of a bias of this magnitude would be to decrease $f_{\text {gas }}$ and $f_{\star}$ by $\sim 8 \%$ and $\sim 3 \%$ respectively, thus decreasing the total baryon fraction by a comparable amount. All other quantitative conclusions in this paper, including the slopes in the gas, stellar, and baryon fraction relations, are robust to this level of uncertainty in the total mass.

Given that our new data set provides the most complete census to date of the hot and cold baryons, the central remaining questions are the detailed properties of lower mass groups and the redshift evolution of the baryonic components. Sanderson et al. (2012) provides a first step towards addressing the former, while we are using the capability of the $H S T$ /WFC3 camera IR channel in conjunction with X-ray data from Chandra and $X M M$ to explore the redshift evolution (programs 12575 and 12634).

The authors thank Alastair Sanderson, Trevor Ponman, Alexie Leauthaud, and Kevin Bundy for extensive discussions pertaining to this project, and are also grateful to Alexie Leauthaud and Yu-Ying Zhang for providing data from their papers. We greatly appreciate the efforts of the anonymous referee, whose suggestions resulted in an improved paper. AHG thanks Carnegie Observatories and IPAC for their hospitality while working on this paper, and acknowledges support from the National Science Foundation through grant NSF-1108957. SS acknowledges the Dunlap Institute for Astronomy and Astrophysics for funding him through the Dunlap Fellowship program. AIZ and DZ thank the MaxPlanck-Institut für Astronomie and the Center for Cosmology and Particle Physics at NYU for their hospitality and support. AIZ also acknowledges support through NASA grants NNX08AX81G and NNX08AC68G associated with XMM observations.

\section{REFERENCES}

Allen, S. W., Rapetti, D. A., Schmidt, R. W., Ebeling, H., Morris, R. G., \& Fabian, A. C. 2008, MNRAS, 383, 879, 0706.0033 Andreon, S. 2010, MNRAS, 407, 263, 1004.2785

Balogh, M. L., McCarthy, I. G., Bower, R. G., \& Eke, V. R. 2008, MNRAS, 385, 1003, 0801.0990

Balogh, M. L., \& McGee, S. L. 2010, MNRAS, 402, L59, 0912.0938

Behroozi, P. S., Wechsler, R. H., \& Conroy, C. 2012, ArXiv e-prints, 1207.6105

Bennett, C. L. et al. 2012, ArXiv e-prints, 1212.5225

Bernardi, M., Hyde, J. B., Sheth, R. K., Miller, C. J., \& Nichol, R. C. 2007, AJ, 133, 1741, arXiv:astro-ph/0607117
Blanton, M. R. et al. 2003, ApJ, 592, 819

Bryan, G. L. 2000, ApJ, 544, L1, arXiv:astro-ph/0009286

Budzynski, J. M., Koposov, S. E., McCarthy, I. G., McGee, S. L. \& Belokurov, V. 2012, MNRAS, 423, 104, 1201.5491

Cappellari, M. et al. 2006, MNRAS, 366, 1126, astro-ph/0505042

- 2011, MNRAS, 413, 813, 1012.1551

- 2012, Nature, 484, 485, 1202.3308

- 2013, MNRAS, 432, 1862, 1208.3523

Carlberg, R. G., Yee, H. K. C., \& Ellingson, E. 1997, ApJ, 478, 462

Cavaliere, A., \& Fusco-Femiano, R. 1978, A\&A, 70, 677 
Christlein, D., \& Zabludoff, A. I. 2003, ApJ, 591, 764, astro-ph/0304031

Conroy, C., Gunn, J. E., \& White, M. 2009, ApJ, 699, 486, 0809.4261

Conroy, C., \& van Dokkum, P. G. 2012, ApJ, 760, 71, 1205.6473

Conroy, C., Wechsler, R. H., \& Kravtsov, A. V. 2007, ApJ, 668, 826, arXiv:astro-ph/0703374

Contini, E., De Lucia, G., Villalobos, A., \& Borgani, S. 2013, MNRAS

Couch, W. J., Balogh, M. L., Bower, R. G., Smail, I., Glazebrook, K., \& Taylor, M. 2001, ApJ, 549, 820, arXiv:astro-ph/0010505

Finn, R. A., Balogh, M. L., Zaritsky, D., Miller, C. J., \& Nichol, R. C. 2008, ApJ, 679, 279, 0802.2282

Giodini, S. et al. 2012, A\&A, 538, A104, 1111.1729

- 2009, ApJ, 703, 982, 0904.0448

Gonzalez, A. H., Zabludoff, A. I., \& Zaritsky, D. 2005, ApJ, 618, 195

Gonzalez, A. H., Zaritsky, D., \& Zabludoff, A. I. 2007, ApJ, 666, $147,0705.1726$

Hyde, J. B., \& Bernardi, M. 2009, MNRAS, 394, 1978, 0810.4922

Komatsu, E. et al. 2011, ApJS, 192, 18, 1001.4538

Kravtsov, A. et al. 2009, in Astronomy, Vol. 2010, astro2010: The Astronomy and Astrophysics Decadal Survey, 164, 0903.0388

Kroupa, P. 2001, MNRAS, 322, 231, arXiv:astro-ph/0009005

Laganá, T. F., Lima Neto, G. B., Andrade-Santos, F., \& Cypriano, E. S. 2008, A\&A, 485, 633, 0804.1102

Laganá, T. F., Zhang, Y.-Y., Reiprich, T. H., \& Schneider, P. 2011, ApJ, 743, 13, 1108.3678

Lau, E. T., Nagai, D., \& Nelson, K. 2013, ArXiv e-prints, 1306.3993

Lauer, T. R. et al. 2007, ApJ, 662, 808, arXiv:astro-ph/0606739

Leauthaud, A. et al. 2012, ApJ, 746, 95, 1109.0010

Lin, Y.-T., Mohr, J. J., \& Stanford, S. A. 2003, ApJ, 591, 749, astro-ph/0304033

- 2004, ApJ, 610, 745, astro-ph/0402308

Lin, Y.-T., Stanford, S. A., Eisenhardt, P. R. M., Vikhlinin, A., Maughan, B. J., \& Kravtsov, A. 2012, ApJ, 745, L3, 1112.1705

Mahdavi, A., Hoekstra, H., Babul, A., Bildfell, C., Jeltema, T., \& Henry, J. P. 2013, ApJ, 767, 116, 1210.3689

Mancone, C., \& Gonzalez, A. H. 2012, PASP, submitted

McCarthy, I. G., Babul, A., Bower, R. G., \& Balogh, M. L. 2008, MNRAS, 386, 1309, 0706.2768

McCarthy, I. G., Schaye, J., Bower, R. G., Ponman, T. J., Booth, C. M., Dalla Vecchia, C., \& Springel, V. 2011, MNRAS, 412, $1965,1008.4799$

McGee, S. L., \& Balogh, M. L. 2010, MNRAS, 403, L79, 0912.3455

McNamara, B. R., \& Nulsen, P. E. J. 2007, ARA\&A, 45, 117, 0709.2152

. 2012, New Journal of Physics, 14, 055023, 1204.0006

Nagai, D., Kravtsov, A. V., \& Vikhlinin, A. 2007, ApJ, 668, 1, arXiv:astro-ph/0703661
Nevalainen, J., David, L., \& Guainazzi, M. 2010, A\&A, 523, A22, 1008.2102

Peebles, P. J. E. 1993, Principles of Physical Cosmology

Planck Collaboration et al. 2013, ArXiv e-prints, 1303.5076

Poggianti, B. M., Bridges, T. J., Komiyama, Y., Yagi, M., Carter, D., Mobasher, B., Okamura, S., \& Kashikawa, N. 2004, ApJ, 601, 197, arXiv:astro-ph/0309449

Puchwein, E., Springel, V., Sijacki, D., \& Dolag, K. 2010, MNRAS, 406, 936, 1001.3018

Purcell, C. W., Bullock, J. S., \& Zentner, A. R. 2007, ApJ, 666, 20, arXiv:astro-ph/0703004

Salpeter, E. E. 1955, ApJ, 121, 161

Sand, D. J. et al. 2011, ApJ, 729, 142, 1011.1310

Sanderson, A. J. R., O'Sullivan, E., Ponman, T. J., Gonzalez, A. H., Sivanandam, S., Zabludoff, A. I., \& Zaritsky, D. 2013, MNRAS, 429, 3288, 1212.1613

Simionescu, A. et al. 2011, Science, 331, 1576, 1102.2429

Sivanandam, S., Zabludoff, A. I., Zaritsky, D., Gonzalez, A. H., \& Kelson, D. D. 2009, ApJ, 691, 1787, 0810.1272

Snowden, S. L., Mushotzky, R. F., Kuntz, K. D., \& Davis, D. S. 2008, A\&A, 478, 615, 0710.2241

Stott, J. P. et al. 2010, ApJ, 718, 23, 1005.4681

Sun, M., Voit, G. M., Donahue, M., Jones, C., Forman, W., \& Vikhlinin, A. 2009, ApJ, 693, 1142, 0805.2320

van Dokkum, P. G., \& Conroy, C. 2012, ApJ, 760, 70, 1205.6471

Vikhlinin, A. et al. 2009, ApJ, 692, 1033, 0805.2207

Vikhlinin, A., Kravtsov, A., Forman, W., Jones, C., Markevitch, M., Murray, S. S., \& Van Speybroeck, L. 2006, ApJ, 640, 691, astro-ph/0507092

Watson, D. F., Berlind, A. A., \& Zentner, A. R. 2012, ApJ, 754, 90, 1201.2407

Weinmann, S. M., van den Bosch, F. C., Yang, X., \& Mo, H. J. 2006, MNRAS, 366, 2, arXiv:astro-ph/0509147

Wetzel, A. R., Tinker, J. L., \& Conroy, C. 2012, MNRAS, 3128, 1107.5311

White, S. D. M., \& Frenk, C. S. 1991, ApJ, 379, 52

Young, O. E., Thomas, P. A., Short, C. J., \& Pearce, F. 2011, MNRAS, 413, 691, 1007.0887

Zandivarez, A., \& Martínez, H. J. 2011, MNRAS, 415, 2553, 1012.3445

Zaritsky, D., Colucci, J. E., Pessev, P. M., Bernstein, R. A., \& Chandar, R. 2012, ArXiv e-prints, 1209.3788

Zhang, Y.-Y., Laganá, T. F., Pierini, D., Puchwein, E., Schneider, P., \& Reiprich, T. H. 2011, A\&A, 535, A78, 1109.0390

Zhang, Y.-Y. et al. 2010, ApJ, 711, 1033, 1001.0780

Zibetti, S., White, S. D. M., Schneider, D. P., \& Brinkmann, J. 2005, MNRAS, 358, 949

\section{APPENDIX}

The Planck first year results were released shortly after submission of this paper (Planck Collaboration et al. 2013). In the paper we discuss the impact on our results of changing from a WMAP7 to Planck cosmology, but retain WMAP7 as the fiducial cosmological model. In this Appendix we also provide versions of the main Tables from the paper for the Planck cosmological parameters. 
TABLE 6

Derived Mass Fractions for Planck Cosmology

$\left(r<r_{500}\right)$

\begin{tabular}{lccc}
\hline \hline Cluster & $f_{\text {gas }}$ & $f_{\text {stellar }}$ & $f_{\text {baryons }}$ \\
& & & \\
\hline Abell 0122 & $0.094 \pm .013$ & $0.026 \pm .003$ & $0.120 \pm .013$ \\
Abell 1651 & $0.139 \pm .013$ & $0.012 \pm .001$ & $0.151 \pm .013$ \\
Abell 2401 & $0.095 \pm .014$ & $0.026 \pm .003$ & $0.121 \pm .014$ \\
Abell 2721 & $0.134 \pm .021$ & $0.016 \pm .002$ & $0.150 \pm .021$ \\
Abell 2811 & $0.132 \pm .011$ & $0.013 \pm .002$ & $0.145 \pm .012$ \\
Abell 2955 & $0.071 \pm .010$ & $0.031 \pm .004$ & $0.103 \pm .011$ \\
Abell 2984 & $0.117 \pm .015$ & $0.041 \pm .005$ & $0.159 \pm .016$ \\
Abell 3112 & $0.142 \pm .010$ & $0.022 \pm .002$ & $0.163 \pm .010$ \\
Abell 3693 & $0.117 \pm .014$ & $0.024 \pm .003$ & $0.141 \pm .014$ \\
Abell 4010 & $0.127 \pm .011$ & $0.023 \pm .003$ & $0.150 \pm .012$ \\
Abell S0084 & $0.094 \pm .012$ & $0.024 \pm .003$ & $0.118 \pm .011$ \\
Abell S0296 & $0.081 \pm .013$ & $0.020 \pm .003$ & $0.101 \pm .014$ \\
\hline Abell 0478 & $0.185 \pm .017$ & - & - \\
Abell 2029 & $0.139 \pm .010$ & - & - \\
Abell 2390 & $0.153 \pm .025$ & - & - \\
& & & \\
\hline
\end{tabular}

Note. - The quoted stellar baryon fractions include a deprojection correction, as discussed in the text.

TABLE 7

Observed Cluster Properties for Planck Cosmology

\begin{tabular}{|c|c|c|c|c|c|c|c|c|c|}
\hline Cluster & $z$ & $\begin{array}{c}T_{X, 2} \\
(\mathrm{keV})\end{array}$ & $\begin{array}{c}L_{B C G+I C L} \\
\left(10^{12} \mathrm{~L}_{\odot}\right)\end{array}$ & $\begin{array}{c}L_{\text {Total }} \\
\left(10^{12} \mathrm{~L}_{\odot}\right)\end{array}$ & $\begin{array}{c}r_{500} \\
(\mathrm{Mpc})\end{array}$ & $\begin{array}{c}M_{500} \\
\left(10^{14} \mathrm{M}_{\odot}\right)\end{array}$ & $\begin{array}{c}M_{g a s, 500} \\
\left(10^{13} \mathrm{M}_{\odot}\right)\end{array}$ & $\begin{array}{l}M_{\star, 2 D, 500} \\
\left(10^{13} \mathrm{M}_{\odot}\right)\end{array}$ & $\begin{array}{c}M_{\star, 3 D, 500} \\
\left(10^{13} \mathrm{M}_{\odot}\right)\end{array}$ \\
\hline Abell 0122 & 0.1134 & $3.65 \pm 0.15$ & $0.91 \pm 0.04$ & $2.87 \pm 0.18$ & $0.93 \pm .03$ & $2.35 \pm .20$ & $2.20 \pm .23$ & $0.76 \pm .05$ & $0.61 \pm .04$ \\
\hline Abell 1651 & 0.0845 & $6.10 \pm 0.25$ & $0.93 \pm 0.09$ & $3.17 \pm 0.23$ & $1.23 \pm .03$ & $5.37 \pm .43$ & $7.45 \pm .35$ & $0.84 \pm .06$ & $0.67 \pm .05$ \\
\hline Abell 2401 & 0.0571 & $2.06 \pm 0.07$ & $0.35 \pm 0.01$ & $1.23 \pm 0.08$ & $0.71 \pm .03$ & $0.99 \pm .11$ & $0.95 \pm .10$ & $0.33 \pm .02$ & $0.26 \pm .02$ \\
\hline Abell 2721 & 0.1144 & $4.78 \pm 0.23$ & $0.61 \pm 0.01$ & $2.82 \pm 0.20$ & $1.07 \pm .03$ & $3.60 \pm .33$ & $4.82 \pm .63$ & $0.75 \pm .05$ & $0.58 \pm .04$ \\
\hline Abell 2811 & 0.1079 & $4.89 \pm 0.20$ & $0.91 \pm 0.15$ & $2.12 \pm 0.18$ & $1.08 \pm .03$ & $3.73 \pm .29$ & $4.94 \pm .19$ & $0.56 \pm .05$ & $0.47 \pm .04$ \\
\hline Abell 2955 & 0.0943 & $2.13 \pm 0.10$ & $0.64 \pm 0.04$ & $1.46 \pm 0.08$ & $0.71 \pm .03$ & $1.03 \pm .12$ & $0.74 \pm .05$ & $0.39 \pm .02$ & $0.32 \pm .02$ \\
\hline Abell 2984 & 0.1042 & $2.08 \pm 0.07$ & $0.92 \pm 0.03$ & $1.79 \pm 0.09$ & $0.70 \pm .02$ & $0.99 \pm .10$ & $1.16 \pm .09$ & $0.48 \pm .02$ & $0.41 \pm .02$ \\
\hline Abell 3112 & 0.0750 & $4.54 \pm 0.11$ & $0.98 \pm 0.05$ & $3.45 \pm 0.23$ & $1.06 \pm .02$ & $3.37 \pm .20$ & $4.77 \pm .18$ & $0.92 \pm .06$ & $0.73 \pm .04$ \\
\hline Abell 3693 & 0.1237 & $3.63 \pm 0.20$ & $0.76 \pm 0.08$ & $2.74 \pm 0.20$ & $0.93 \pm .03$ & $2.36 \pm .24$ & $2.76 \pm .16$ & $0.73 \pm .05$ & $0.57 \pm .04$ \\
\hline Abell 4010 & 0.0963 & $3.78 \pm 0.13$ & $0.87 \pm 0.13$ & $2.68 \pm 0.21$ & $0.95 \pm .02$ & $2.51 \pm .19$ & $3.18 \pm .13$ & $0.71 \pm .06$ & $0.57 \pm .05$ \\
\hline Abell S0084 & 0.1100 & $3.75 \pm 0.20$ & $0.77 \pm 0.03$ & $2.78 \pm 0.19$ & $0.94 \pm .03$ & $2.47 \pm .25$ & $2.32 \pm .17$ & $0.74 \pm .05$ & $0.58 \pm .04$ \\
\hline Abell S0296 & 0.0696 & $2.70 \pm 0.21$ & $0.61 \pm 0.02$ & $1.38 \pm 0.08$ & $0.81 \pm .04$ & $1.51 \pm .21$ & $1.22 \pm .11$ & $0.37 \pm .02$ & $0.31 \pm .01$ \\
\hline Abell 0478 & 0.0881 & $7.09 \pm 0.12$ & - & - & $1.33 \pm 0.03$ & $6.80 \pm 0.39$ & $12.6 \pm 0.9$ & & \\
\hline Abell 2029 & 0.0773 & $8.41 \pm 0.12$ & - & - & $1.47 \pm 0.03$ & $9.01 \pm 0.57$ & $12.5 \pm 0.4$ & 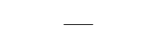 & - \\
\hline Abell 2390 & 0.2329 & $10.6 \pm 0.8$ & - & - & $1.53 \pm 0.08$ & $12.1 \pm 1.8$ & $18.5 \pm 1.2$ & - & 一 \\
\hline
\end{tabular}

NOTE. - Abell 0478, Abell 2029, and Abell 2390 are not part of the main sample. These clusters were included only in the X-ray analysis to extend the baseline to higher mass, but have no photometry equivalent to the other systems with which to measure the stellar mass. At the high mass end; however, the stellar component contributes a relatively small fraction of the total baryons. The luminosities include appropriate $\mathrm{e}+\mathrm{k}$ corrections for each galaxy from GZZ07. The stellar masses are quoted as observed, with no deprojection correction applied. 
TABLE 8

Derived $M_{j}-M_{500}$ Relations for Planck COSMOLOGY

\begin{tabular}{lcr}
\hline \hline Component & $a$ & $b$ \\
\hline$M_{\star, 2 D}$ & $3.9 \pm 0.2 \times 10^{-2}$ & $0.56 \pm 0.05$ \\
$M_{\star, 3 D}$ & $3.3 \pm 0.2 \times 10^{-2}$ & $0.53 \pm 0.05$ \\
$M_{\text {gas }}$ & $9.2 \pm 0.4 \times 10^{-2}$ & $1.26 \pm 0.03$ \\
$M_{\text {bary }}$ & $1.21 \pm 0.05 \times 10^{-1}$ & $1.17 \pm 0.04$ \\
\hline$M_{\star, 2 D} / M_{\text {gas }}$ & $4.70 \pm 0.03 \times 10^{-1}$ & $-0.85 \pm 0.05$ \\
$M_{\star, 3 D} / M_{\text {gas }}$ & $3.87 \pm 0.02 \times 10^{-1}$ & $-0.81 \pm 0.05$ \\
\hline
\end{tabular}

Note. - Best fit parameters for the relation $M_{j}=$ $a\left(M_{500} / 10^{14} M_{\odot}\right)^{b}$, where $M_{j}$ is the mass contained in each baryonic component. $M_{\text {bary }}$ is derived using the deprojected stellar mass. The slope for the baryon fraction relations is equivalent to $1-b$. We also include the best fit parameters for the stellar-to-gas mass ratios as a function of $M_{500}$. The relation for the gas mass is derived including the clusters from Vikhlinin et al. (2006), while the other relations are derived using only clusters with both gas and stellar mass data. 